Geometry \& Topology

Volume 2 (1998) 31-64

Published: 21 March 1998

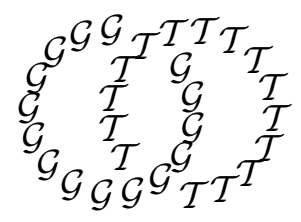

\title{
A natural framing of knots
}

\author{
Michael T Greene \\ Bert Wiest \\ Mathematics Institute \\ University of Warwick \\ Coventry CV4 7AL, UK \\ Email: mtg@maths.warwick.ac.uk or mtg@uk.radan.com \\ bertold@maths.warwick.ac.uk
}

\begin{abstract}
Given a knot $K$ in the 3 -sphere, consider a singular disk bounded by $K$ and the intersections of $K$ with the interior of the disk. The absolute number of intersections, minimised over all choices of singular disk with a given algebraic number of intersections, defines the framing function of the knot. We show that the framing function is symmetric except at a finite number of points. The symmetry axis is a new knot invariant, called the natural framing of the knot. We calculate the natural framing of torus knots and some other knots, and discuss some of its properties and its relations to the signature and other well-known knot invariants.
\end{abstract}

AMS Classification numbers Primary: 57M25

Secondary: 20F05

Keywords: Knot, link, knot invariant, framing, natural framing, torus knot, Cayley graph

Proposed: Cameron Gordon

Seconded: Joan Birman, Walter Neumann
Received: 4 August 1997

Accepted: 19 March 1998

\section{Copyright Geometry and Topology}


Let $K: S^{1} \rightarrow S^{3}$ be an unoriented knot. Let $D$ be the 2 -disk. We define a compressing disk of $K$ to be a map $f: D \rightarrow S^{3}$ such that $\left.f\right|_{\partial D}=K$ and such that $\left.f\right|_{\operatorname{int}(D)}$ is transverse to $K$. Then $\left.f\right|_{\operatorname{int}(D)}$ has only finitely many intersections with the knot. We call the intersection points the holes of the compressing disk, and denote their number by $n(f)$. So $n(f)=\mid\left\{f^{-1}(K) \cap\right.$ $\operatorname{int}(D)\} \mid$. One rather crude invariant of the knot $K$ is the knottedness

$$
L(K):=\min \{n(f) \mid f \text { a compressing disk }\},
$$

which was first considered in [9]. (The English term 'knottedness' was taken from [1].)

We note that holes can occur with two different signs (depending on the direction in which $K$ pierces $f(D)$ ), so we can refine the above invariant by defining the framing function $n_{K}: \mathbb{Z} \rightarrow \mathbb{N}$ as follows: for a given $k \in \mathbb{Z}$ we minimize the absolute number of holes among all compressing disks with algebraically $k$ holes. We shall see that (except at finitely many points in $\mathbb{Z}$ ) this function is symmetric around some value of $k$, and we call this 'asymptotic symmetry axis' $k$ the natural framing of $K$. The aim of this paper is to determine the natural framing of certain classes of knot, and to study its properties and relations with other knot invariants.

In section 1 we define the natural framing of knots and show that it shares many properties with the signature. In section 2 we define the natural framing of each component of a link, and calculate it for a number of links. It seems that for most "simple" knots the natural framing number is even; in section 3, however, we show that knots with odd natural framing do exist. In section 4 we prove that the natural framing of the $(p, q)$-torus knot is $-(p-1)(q-1)$. In section 5 we summarize all the information we have about the natural framing of knots with up to seven crossings.

\section{Definitions and general results}

Let $K: S^{1} \rightarrow S^{3}$ be a knot, and let $f: D \rightarrow S^{3}$ be a compressing disk. By a standard general position argument we can assume that the singularities of a compressing disk $f$ are all transverse self-intersectons which may end either in $K$ or in the branch points of Whitney umbrellas (for details see [11]). In what follows, we will, without further explanation, talk about the double lines or the Whitney umbrellas of a compressing disk. The following result is from [9]. 
Lemma 1.1 Let $f: D \rightarrow S^{3}$ be a compressing disk. Then there exists a compressing disk $f^{\prime}: D \rightarrow S^{3}$, where $f^{\prime}$ is an immersion, such that $n\left(f^{\prime}\right) \leqslant$ $n(f)$.

Proof We homotope $f$ into general position. Now we only have to get rid of Whitney umbrellas. The double line starting at a Whitney umbrellas either ends in another Whitney umbrella or in $K$. We move the branch point along the double line, shrinking the double line until it doesn't contain triple points. In the first case we then perform a surgery along the double line; this eliminates the double line and the two Whitney umbrellas. In the second case we slide the knot over the branch point of the Whitney umbrella; this leaves the knot type unchanged, and eliminates the Whitney umbrella, the double line, and one hole. (Also, it changes the framing represented by the compressing disk by \pm 1 .) Applying this process to every branch point gives the result.

Proposition 1.2 (Pannwitz) For any knot $K, L(K)$ is even.

Proof Let $f: D \rightarrow S^{3}$ be a compressing disk with $n(f)=L(K)$. As we have just seen, we may assume that $f$ is an immersion, so it has no Whitney umbrellas. Every hole of $f$ is the beginning of a double point line. Now this line must end in another hole (not a Whitney umbrella); so there must be an even number of holes.

Remark For any non-trivial knot $K$ we have $L(K) \geqslant 2$. This follows from Dehn's Lemma and the fact that $L(K)$ is even.

Theorem 1.3 The knottedness is additive under connected sum, ie if $K_{1}$ and $K_{2}$ are knots then $L\left(K_{1} \# K_{2}\right)=L\left(K_{1}\right)+L\left(K_{2}\right)$.

Proof It is obvious that $L\left(K_{1} \# K_{2}\right) \leqslant L\left(K_{1}\right)+L\left(K_{2}\right)$; we need to prove the opposite inequality.

Choose an embedded sphere $S$ in $S^{3}$ which intersects the knot in only two points, splitting $K_{1}$ from $K_{2}$. Let $f: D \rightarrow S^{3}$ be a compressing disk of $K_{1} \# K_{2}$ with only $L\left(K_{1} \# K_{2}\right)$ holes. Make the map $f$ transverse to $S$ without changing the number of holes. $f^{-1}(S)$ consists of one arc $A$ in $D$ connecting two points on $\partial D$, and a number of disjoint circles in $D$. Choose an outermost one of those, and call it $c$. Let $C$ be the disk bounded by $c$. Say $c$ represents an element $[k] \in H_{1}(S \backslash(S \cap K)) \cong H_{1}\left(S^{3} \backslash\left(K_{1} \# K_{2}\right)\right) \cong \mathbb{Z}$; then there are at least $k$ holes inside $C . S \backslash(S \cap K)$ is a sphere with two holes, so we can replace $\left.f\right|_{C}$ 
by a map whose image lies entirely in $S$, and which has precisely $k$ intersections with $K$. After pushing the image away from $S$ by a small homotopy, we have a map $f^{\prime}$ such that $\left.f^{\prime}\right|_{C}$ has precisely $k$ holes and maps no point of $C$, or a small neighbourhood of $C$, to $S$.

This construction has replaced the compressing disk $f$ by a compressing disk $f^{\prime}$ with at most as many holes (and the same framing). Since by hypothesis $n(f)$ is minimal, we have $n\left(f^{\prime}\right)=n(f)$. Applying the construction to all outermost circles of intersection yields a compressing disk with $L\left(K_{1} \# K_{2}\right)$ holes, which has the property that the arc $A$ is mapped to $S$, one of the two components of $D \backslash A$ is entirely mapped to one of the two components of $S^{3} \backslash S$, and the other component of $D \backslash A$ is mapped to the other component of $S^{3} \backslash S$. This gives rise to compressing disks for the knots $K_{1}$ and $K_{2}$ with $n_{1}$ and $n_{2}$ holes respectively such that $n_{1}+n_{2}=L\left(K_{1} \# K_{2}\right)$.

Let $d$ be the inner boundary of a small neighbourhood of $\partial D$ in $D$; then $d$ is a curve in $D$ 'close to $\partial D$ '. $f$ maps $d$ to a longitude of the knot. If we orient $d$ and $\partial D$ in the same way, then $f(d)$ and $f(\partial D)=K$ have a linking number $[f(d)] \in H_{1}\left(S^{3} \backslash K\right) \cong \mathbb{Z}$. We denote this number by $k(f)$. So $d$ represents the framing $k(f)$ of $K$. Geometrically, this framing can be obtained as follows: choose an orientation of $D$; this determines an orientation of $\partial D$ and hence of $K$. For $i \in\{1, \ldots, n(f)\}$ let $x_{i} \in D$ be the $i$ th hole of $f$. Let $\sigma(i)=1$ if a positive basis of $T_{f\left(x_{i}\right)} f(D)$ followed by a positive tangent vector of $K$ at $f\left(x_{i}\right)$ forms a positive basis of $T_{f\left(x_{i}\right)} S^{3}$, and let $\sigma(i)=-1$ otherwise. Then $k(f)=\sum_{i=1}^{n(f)} \sigma(i)$.

To every knot $K$ we can associate a function $n_{K}: \mathbb{Z} \rightarrow \mathbb{N}$ which we call the framing function as follows:

$$
n_{K}\left(k^{\prime}\right):=\min \left\{n(f) \mid f \text { a compressing disk with } k(f)=k^{\prime}\right\}
$$

Notice that $L(K)=\min n_{K}$.

Proposition 1.4 For any knot $K$, the function $n_{K}$ has the following properties.

(i) $n_{K}(k) \geqslant|k|$ for all $k \in \mathbb{Z}$.

(ii) $n_{K}$ maps even numbers to even numbers and odd numbers to odd numbers.

(iii) 'Continuity': For any $k \in \mathbb{Z}$ we have $n_{K}(k+1)=n_{K}(k) \pm 1$. 
(iv) If $k \in \mathbb{Z}$ is odd, then $n_{K}(k)=\min \left\{n_{K}(k-1), n_{K}(k+1)\right\}+1$. In particular, the function $n_{K}$ is completely determined by its values on even numbers.

Proof (i) is obvious.

(ii) follows from the fact that $\sum_{i=1}^{n} \sigma(i)$ (where $\sigma(i) \in\{-1,1\}$ ) is even if and only if $n$ is even.

(iii) By (ii), $n_{K}(k+1) \neq n_{K}(k)$. So for definiteness say $n_{K}(k+1)>n_{K}(k)$. Let $f$ be a compressing map with $n_{K}(k)$ holes and framing $k$. By artificially introducing a Whitney umbrella we can obtain a compressing disk with $n_{K}(k)+1$ holes and framing $k+1$.

(iv) Suppose $f$ is a compressing disk with odd framing number. Then $f$ has at least one Whitney umbrella. If we remove this as in Lemma 1.1, we obtain another compressing disk with framing number $k(f) \pm 1$ and $n(f)-1$ holes. It follows that for every odd $z \in \mathbb{Z}$ we have either $n_{K}(z+1)<n_{K}(z)$ or $n_{K}(z-1)<n_{K}(z)$. This implies (iv).

Let $B$ in $S^{3}$ be a double-point line of a compressing disk $f$. Suppose that $B$ does not end in a Whitney umbrella, and suppose $B$ is not a closed curve. Then $f^{-1}(B)$ consists of two lines in $D$ (not necessarily disjoint or embedded). There are two possible cases: either one of them connects two holes and the other connects two points on $\partial D$, when $B$ is a ribbon singularity; or each of the two lines connects one hole with one point on $\partial D$, when $B$ is a clasp singularity. We call a clasp singularity positive or negative if it ends at two positive or negative holes, respectively. We say a clasp or ribbon singularity is short if its double-point line meets no triple points.

Conjecture For any nontrivial knot $K$ we have $n_{K}(0) \geqslant 4$.

This is a strengthened form of Dehn's Lemma. Dehn's Lemma states that the only knot in $S^{3}$ which has a compressing disk without holes is the unknot. Our conjecture asserts that this remains true under the weakened hypothesis that the compressing disk has 2 intersections with $K$ of opposite sign (ie one ribbon singularity $B$ ). This is easy to show with the extra hypothesis that $B$ is short.

Definition 1.5 The function $n_{K}$ gives rise to a framing of the knot:

$$
\nu(K):=\lim _{k \rightarrow \infty} \frac{n_{K}(-k)-n_{K}(k)}{2}
$$

is called the natural framing of $K$. Alternatively, we can define $\nu(K)$ to be the unique integer such that there exists an $N \in \mathbb{N}$ with $n_{K}(\nu(K)-k)=$ $n_{K}(\nu(K)+k)$ for all $k \geqslant N$. 
Lemma 1.6 This is well-defined.

Proof For $k \in \mathbb{N}$ let $a_{k}=n_{K}(-k)-|-k|$ and let $b_{k}=n_{K}(k)-|k|$. We have $\left(n_{K}(-k)-n_{K}(k)\right) / 2=\left(a_{k}-b_{k}\right) / 2$. Moreover, by Proposition 1.4 (i) and (iii), both $a_{k}$ and $b_{k}$ are decreasing sequences in $2 \mathbb{N}$. Therefore there exists an $N \in \mathbb{N}$ such that for $k \geqslant N$ the sequences $\left(a_{k}\right)$ and $\left(b_{k}\right)$ are constant. It follows that the limit exists. Furthermore, by 1.4 (ii) we have that $n_{K}(-k)-n_{K}(k)$ is even for all $k \in \mathbb{N}$, so $\nu(K)$ is indeed an integer.

The natural framing is an 'asymptotic symmetry axis' of the framing function. As a first example, we look at the framing function of the figure-of-eight knot $4_{1}$ (from the table in [10]). Only the value $n_{4_{1}}(0)=4$ is conjectured. The fact

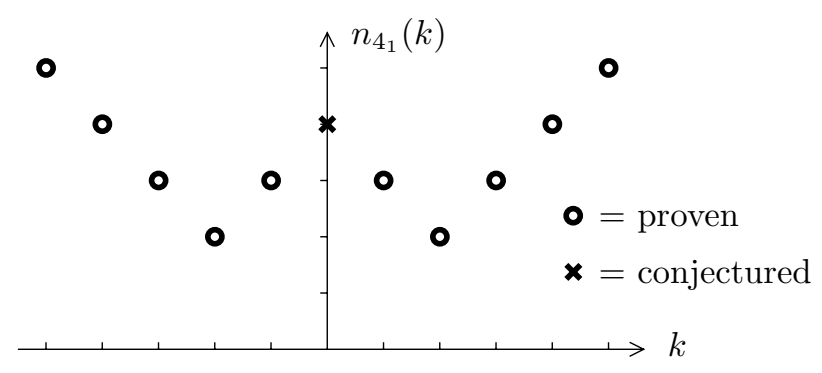

Figure 1: The function $n_{4_{1}}$

that $n_{4_{1}}(2)=2$ follows from an easy construction (a compressing disk with one positive clasp and no other singularities). Similarly we see that $n_{4_{1}}(-2)=2$. The rest of the proof follows immediately from Proposition 1.4. We observe that $\nu\left(4_{1}\right)=0$. More generally we have:

Proposition 1.7 Let $K$ be a knot, and let $m K$ be its mirror image. Then $n_{K}(k)=n_{m K}(-k)$ for all $k \in \mathbb{Z}$. In particular, $\nu(K)=-\nu(m K)$, and if $K$ is amphicheiral then $\nu(K)=0$.

Proof Let $f$ be a compressing disk with framing $k$ and with $n_{K}(k)$ holes. Then $m \circ f$, where $m$ is the mirror map, is a compressing map of the knot $m K$ with framing $-k$ and also $n_{K}(k)$ holes. It follows that $n_{m K}(-k) \leqslant n_{K}(k)$. The opposite inequality is proved in the same way.

So the natural framing $\nu(K)$ changes sign under taking the mirror image, a property it shares with the well-known signature $\sigma(K)$ (see, for example, [10]). There is an even closer relation: 
Proposition 1.8 If a knot $K$ has a compressing disk with positive clasps and closed double-point lines, but no negative clasps or ribbons, then $\nu(K) \geqslant 0$. If all the clasps are short, then we have in addition $\sigma(K) \leqslant 0$.

Proof If there exists a compressing disk with say $c$ positive clasps and no negative clasps or ribbons then we have $n_{K}(k)=k$ for all $k \geqslant 2 c$. It follows that $\nu(K) \geqslant 0$. If in addition all these clasps are short, then we can unknot $K$ by $c$ negative crossing changes. According to [3] it follows that $-2 c \leqslant \sigma(K) \leqslant 0$.

As an application, we can prove that for a large family of knots the natural framing and the signature are both zero. We only have to construct a compressing disk with only short positive clasps and no other singularities, and another compressing disk with only short negative clasps.

Examples (1) If $K$ is one of the so-called 'twist knots' $4_{1}, 6_{1}, 8_{1}, 10_{1}$ etc then $\nu(K)=\sigma(K)=0$. The case of the stevedore's knot $6_{1}$ is illustrated in Figure 2 , and the other cases are similar. On the left we see a compressing disk with
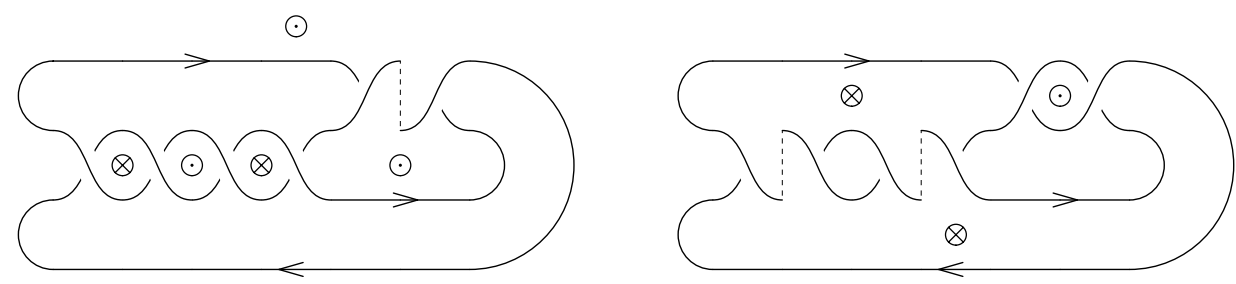

Figure 2: Two different compressing disks of the knot $6_{1}$

one short clasp singularity with positive sign (indicated by the dashed lines), proving that $n_{K_{4}}(2) \leqslant 2$. On the right we see a compressing disk with two short clasp singularities of negative sign, proving that $n_{K_{4}}(-4) \leqslant 4$.

(2) This construction can be generalized. Consider the family of $r$-bridge knots indicated in Figure 3, where the $a_{j}^{(i)}$ and $b_{j}^{(i)}$ are all non-negative and even. On the right, choosing a particular 12-crossing knot as an example, we see two compressing disks; the first has only short positive clasps, the second only short negative clasps.

Conjecture The natural framing of the twist knots $3_{1}, 5_{2}, 7_{2}, 9_{2}, \ldots$ is 2 . More precisely, we conjecture that for such knots $n(k)=2+|k-2|$. The values for $k>0$ are easy to prove; the values for $k \leqslant 0$ seem hard. 

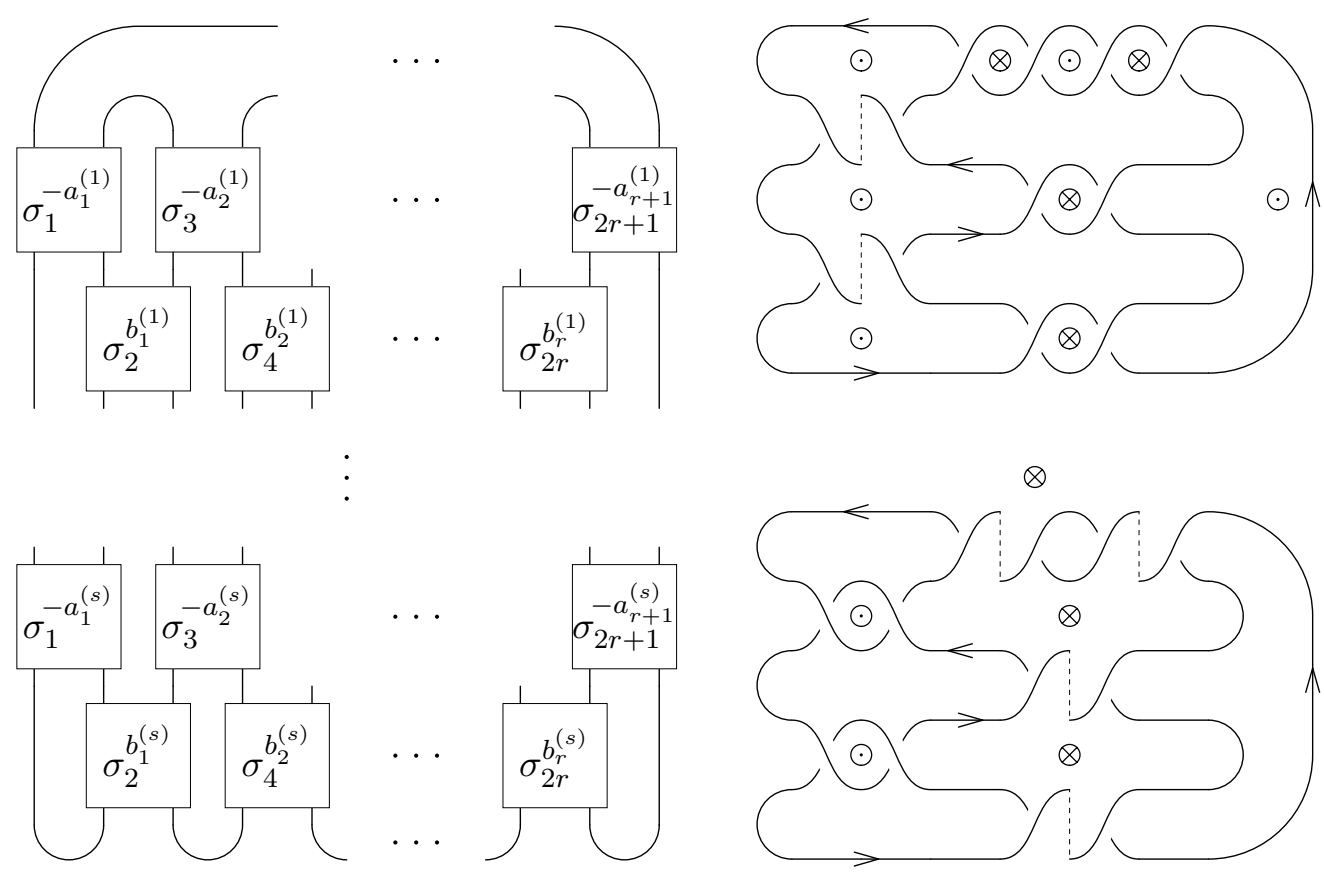

Figure 3: A family of knots whose natural framing and signature are zero

Even more generally, we can consider the $\frac{4 m l+1}{2 l}$-two-bridge knot (in the notation of [1], Chapter 12). This is the rational knot $C(2 m 2 l)$ in the notation of Conway [2], and for $l=1$ we get twist knots. We conjecture that for $m, l \in \mathbb{Z}^{+}$ the natural framing of this knot is $\min (2 m, 2 l)$.

Lemma 1.9 Let $K_{1}$ and $K_{2}$ be knots. Then

$$
n_{K_{1} \# K_{2}}(k)=\min _{k^{\prime} \in \mathbb{Z}}\left(n_{K_{1}}\left(k^{\prime}\right)+n_{K_{2}}\left(k-k^{\prime}\right)\right) .
$$

Proof As in the proof of Theorem 1.3 we see that for every compressing disk $f$ of $K_{1} \# K_{2}$ with framing $k$ and $n_{K_{1} \# K_{2}}(k)$ holes we can find another compressing disk $f^{\prime}$ with the same framing, the same number of holes and only one intersection curve with a separating sphere. The result follows.

Proposition 1.10 The natural framing is additive under connected sum; ie if $K_{1}$ and $K_{2}$ are knots then $\nu\left(K_{1} \# K_{2}\right)=\nu\left(K_{1}\right)+\nu\left(K_{2}\right)$.

Proof There exist $N, M \in \mathbb{N}$ and $c_{1}, c_{2} \in \mathbb{Z}$ such that $n_{K_{1}}\left(\nu\left(K_{1}\right)+k\right)=c_{1}+|k|$ and $n_{K_{2}}\left(\nu\left(K_{2}\right)+l\right)=c_{2}+|l|$ for all $k, l \in \mathbb{Z}$ with $|k| \geqslant N$ and $|l| \geqslant M$. 
Now let $k \geqslant N$ and $l \geqslant M$. By Lemma 1.9 we have

$$
\begin{aligned}
n_{K_{1} \# K_{2}}\left(\nu\left(K_{1}\right)+\nu\left(K_{2}\right)+k+l\right) & \leqslant n_{K_{1}}\left(\nu\left(K_{1}\right)+k\right)+n_{K_{2}}\left(\nu\left(K_{2}\right)+l\right) \\
& =c_{1}+k+c_{2}+l .
\end{aligned}
$$

Furthermore we have $n_{K_{1}}\left(\nu\left(K_{1}\right)+k+a\right) \geqslant c_{1}+k+a$ and $n_{K_{2}}\left(\nu\left(K_{2}\right)+l-a\right) \geqslant$ $c_{2}+l-a$ for all $a \in \mathbb{Z}$. Lemma 1.9 implies that

$$
\begin{aligned}
& n_{K_{1} \# K_{2}}\left(\nu\left(K_{1}\right)+\nu\left(K_{2}\right)+k+l\right)=\min _{a \in \mathbb{Z}}\left(n_{K_{1}}\left(\nu\left(K_{1}\right)+k+a\right)\right. \\
& \geqslant c_{1}+k+c_{2}+l \text {. } \\
& \left.+n_{K_{2}}\left(\nu\left(K_{2}\right)+l-a\right)\right)
\end{aligned}
$$

We have proved that $n_{K_{1} \# K_{2}}\left(\nu\left(K_{1}\right)+\nu\left(K_{2}\right)+k\right)=c_{1}+c_{2}+|k|$ for all $k \in \mathbb{Z}$ with $k \geqslant N+M$. The case $k \leqslant-N-M$ is proved similarly.

\section{A natural framing of links}

In this section we define a natural framing for each component of a link. By giving an example we prove that these framing numbers are not always even, and that they are not determined by the natural framings of the individual link components (regarded as knots) and their linking numbers.

Let $L=L_{1} \cup \ldots \cup L_{m}: S^{1} \cup \ldots \cup S^{1} \rightarrow S^{3}$ be an unoriented link with $m$ components. Let $D$ be the 2 -disk. We define a compressing disk of the $i$ th link component $L_{i}(i \in\{1, \ldots, m\})$ to be a map $f: D \rightarrow S^{3}$ transverse to $L$ such such that $\left.f\right|_{\partial D}=L_{i}$. Then $\left.f\right|_{\operatorname{int}(D)}$ has only finitely many intersections with $L$. We call these intersection points the holes of the compressing disk, and denote their number by $n(f)$. We choose an orientation of $L_{i}$. This induces an orientation of $D$. We look at an intersection point of $\left.f\right|_{\operatorname{int}(D)}$ with $L_{i}$. We define such a hole to be positive or negative, depending on whether a positive basis of the tangent space to $D$ followed by a positive tangent vector to $L_{i}$ forms a positive or a negative basis of $S^{3}$, respectively. This is well-defined (ie independent of the choice of orientation of $\left.L_{i}\right)$. We denote by $k(f) \in \mathbb{Z}$ the number of intersections of $\left.f\right|_{\operatorname{int}(D)}$ with $L_{i}$ (not all link components!), counted algebraically. Again, we can think of $k(f)$ as the framing of $L_{i}$ defined by $f$.

To every component $L_{i}$ of $L$ we can associate a function $n_{i}: \mathbb{Z} \rightarrow \mathbb{N}$ which we call the $i$ th framing function as follows:

$$
n_{i}\left(k^{\prime}\right):=\min \left\{n(f) \mid f \text { a compressing disk of } L_{i} \text { with } k(f)=k^{\prime}\right\} .
$$


Precisely as in the case of knots we define the natural framing of the component $L_{i}$ of $L$ by

$$
\nu_{i}(L):=\lim _{k \rightarrow \infty} \frac{n_{i}(-k)-n_{i}(k)}{2},
$$

and prove that this limit exists. It is clear from the definition that the natural framing of each component of $L$ is an integer multiple of $\frac{1}{2}$. We claim:

Lemma 2.1 The natural framing of each component of $L$ is an integer.

Proof It suffices to show that for each $i \in\{1, \ldots, m\}$ we have either $n_{i}(k) \equiv k$ $(\bmod 2)$ for all $k \in \mathbb{Z}$ or $n_{i}(k)+1 \equiv k(\bmod 2)$ for all $k \in \mathbb{Z}$. To see this, let $f$ be a compressing disk with $n(f)=n\left(k^{\prime}\right)$, where $k^{\prime}=k(f)$. Equip all link components with an orientation, no matter which. Let $\tilde{k}(f)$ be the number of intersections of $\left.f\right|_{\operatorname{int}(D)}$ with all components of $L$, counted algebraically. We have

$$
\tilde{k}(f) \equiv n(f)(\bmod 2)
$$

and

$$
k(f)=\tilde{k}(f)-\sum_{j \neq i} l k\left(L_{i}, L_{j}\right)
$$

It follows that

$$
n(f)-k(f) \equiv \sum_{j \neq i} l k\left(L_{i}, L_{j}\right)(\bmod 2) .
$$

Since $\sum_{j \neq i} l k\left(L_{i}, L_{j}\right)$ is independent of $f$, the result follows.

Examples (1) The trivial link on $m$ components. Let $L$ be the link consisting of $m$ unknotted, unlinked components. It is easy to see that the framing function of each component is $n_{i}(k)=|k|$. It follows that $\nu_{i}(L)=0$ for each $i \in\{1, \ldots, m\}$.

(2) The Hopf link. The two link components $L_{1}$ and $L_{2}$ have linking number 1 , so any compressing disk of the first component has at least one intersection with the second. Therefore we have $n_{1}(k) \geqslant|k|+1$ for all $k \in \mathbb{Z}$. It is easy to construct compressing disks of $L_{1}$ which have framing $k$ and $|k|+1$ holes, so $n_{1}(k)=|k|+1$ for all $k \in \mathbb{Z}$. It follows that $\nu_{1}(L)=0$, and similarly $\nu_{2}(L)=0$.

(3) The link with two components shown in Figure 4 for any $t \geqslant 1$, consisting of an unknot and a twist-knot (eg for $t=2$ we get the knot $6_{1}$ ). 

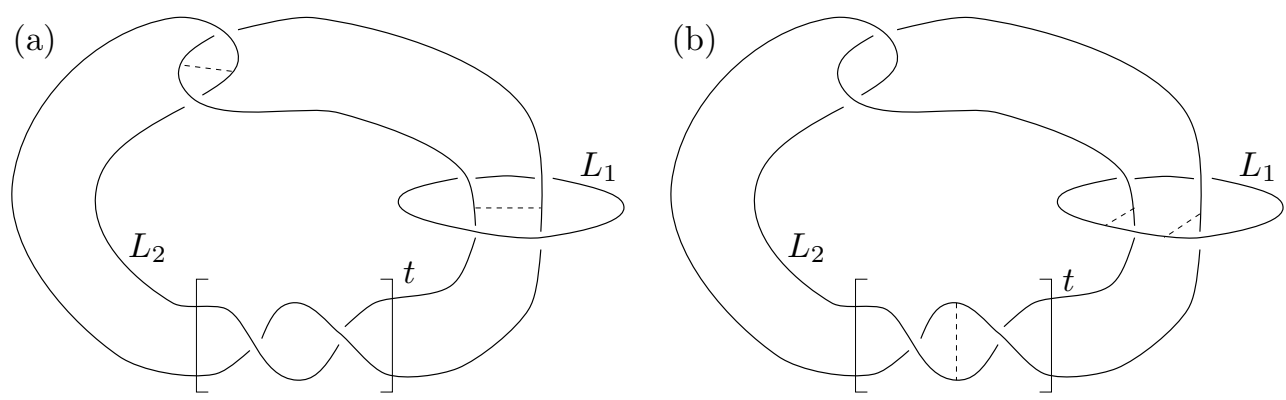

Figure 4: Two different compressing disks of $L_{2}$

Proposition 2.2 We have $\nu_{1}(L)=0$ and $\nu_{2}(L)=1$

Proof $L_{1}$, regarded only as a closed curve in $S^{3} \backslash L_{2}$, represents a nontrivial element of $\pi_{1}\left(S^{3} \backslash L_{2}\right)$. Therefore any compressing disk of $L_{1}$ has at least one intersection with $L_{2}$. Since the linking number of $L_{1}$ and $L_{2}$ is 0 , there must in fact be a minimum of 2 holes. Therefore we have $n_{1}(k) \geqslant|k|+2$ for $k \in \mathbb{Z}$, and again equality follows by construction. It follows that $\nu_{1}(L)=0$.

In order to visualize compressing disks of $L_{2}$, we draw their lines of selfintersections, and also the lines of intersection with the obvious compressing disk of $L_{1}$ which has no self-intersections and two intersection points with $L_{2}$.

There exists a compressing disk of $L_{2}$, indicated in Figure 4(a), which is disjoint from $L_{1}$, and has one clasp singularity connecting two positive holes. This proves that $n_{2}(k)=k$ for $k \geqslant 2$.

On the other hand, there exists a compressing disk, indicated in Figure 4(b), which has two intersections of opposite sign with $L_{1}$, and $t$ clasp singularities, each connecting two negative holes. This proves that $n_{2}(k) \leqslant|k|+2$ for $k \leqslant-2 t$.

Next let $f$ be a compressing disk with $k(f) \leqslant 0$. The image of $f$ has an even number of intersections with $L_{1}$, because the linking number of $L_{1}$ with $L_{2}$ is 0 . We distinguish two cases. If $f$ is disjoint from $L_{1}$, ie if the image of $f$ is contained in the solid torus $S^{3} \backslash L_{1}$, then using a simple covering space argument it is easy to prove that $f$ has at least two positive holes. So $n(f) \geqslant|k(f)|+4$ for all such compressing disks. If, however, the image of $f$ has $2 s$ intersections with $L_{1}(s \geqslant 1)$ then $n(f) \geqslant|k(f)|+2 s$. In either case, $n(f) \geqslant|k(f)|+2$. It follows that $n_{2}(k) \geqslant|k|+2$ for $k \leqslant 0$.

Altogether we have $n_{2}(k)=|k|+2$ for $k \leqslant-2 t$, and therefore $\nu_{2}(L)=1$. 
Example (4) The Whitehead link, which is the case $t=0$ in Figure 4. We have $\nu_{2}(L)=1$, by precisely the same argument as in the case $t>0$. The Whitehead link is isotopic to itself with the roles of $L_{1}$ and $L_{2}$ interchanged. Therefore $\nu_{1}(L)=1$. (Note that the reasoning behind the calculation of $\nu_{1}(L)$ for the case $t>0$ does not apply here, since the path $L_{1}$ is contractible in $\left.S^{3} \backslash L_{2}.\right)$

These results are remarkable, because they show that $\nu_{i}(L)$, the natural framing of the $i$ th link component, is not determined by the natural framing numbers of all individual link components and their linking numbers. More precisely, $\nu_{i}(L)$ is not determined by $\nu\left(L_{1}\right), \ldots, \nu\left(L_{m}\right)$ and $l k\left(L_{r}, L_{s}\right)(r, s \in\{1, \ldots, m\})$. Also, the natural framing numbers of link components can be odd. It is not obvious that knots with odd natural framings exist, but example (3) will lead to the construction of such a knot in the next section.

Proposition 2.3 Let $L^{(1)}=L_{1}^{(1)} \cup \ldots \cup L_{r}^{(1)}$ and $L^{(2)}=L_{1}^{(2)} \cup \ldots \cup L_{s}^{(2)}$ be links in $S^{3}$ with $r$ and $s$ components, respectively. Let $L^{(3)}$ be the link obtained by embedding $L^{(1)}$ and $L^{(2)}$ on either side of some embedded $S^{2}$ in $S^{3}$, and connecting $L_{1}^{(1)}$ and $L_{1}^{(2)}$ by a 'band', as in the construction of the connected sum of two knots. $L^{(3)}$ has $r+s-1$ components, which we label such that $L_{1}^{(3)}$ is the one that contains the 'band'. Then $\nu_{1}\left(L^{(3)}\right)=\nu_{1}\left(L^{(1)}\right)+\nu_{1}\left(L^{(2)}\right)$.

Proof The proof is virtually identical to the proof of 1.10. (Note that the link $L^{(3)}$ does not depend on the choice of the band.)

Corollary 2.4 Let $L=L_{1} \cup \ldots \cup L_{r}$ be a link in $S^{3}$. Then we can add one unknotted link component to $L$ in such a way that the natural framing of $L_{1}$ is increased by 1 , and such that the natural framings of $L_{2}, \ldots, L_{r}$ remain unchanged.

Proof Both components of the Whitehead link are unknotted, so taking the connected sum of $L_{1}$ with either of its components doesn't change the type of $L$. The result now follows from Example (4) and Proposition 2.3.

\section{$3 \quad$ A knot with odd natural framing}

For 'simple' knots, eg knots with low crossing number, the natural framing always appears to be an even number. In this section we exhibit a knot $K$ with 


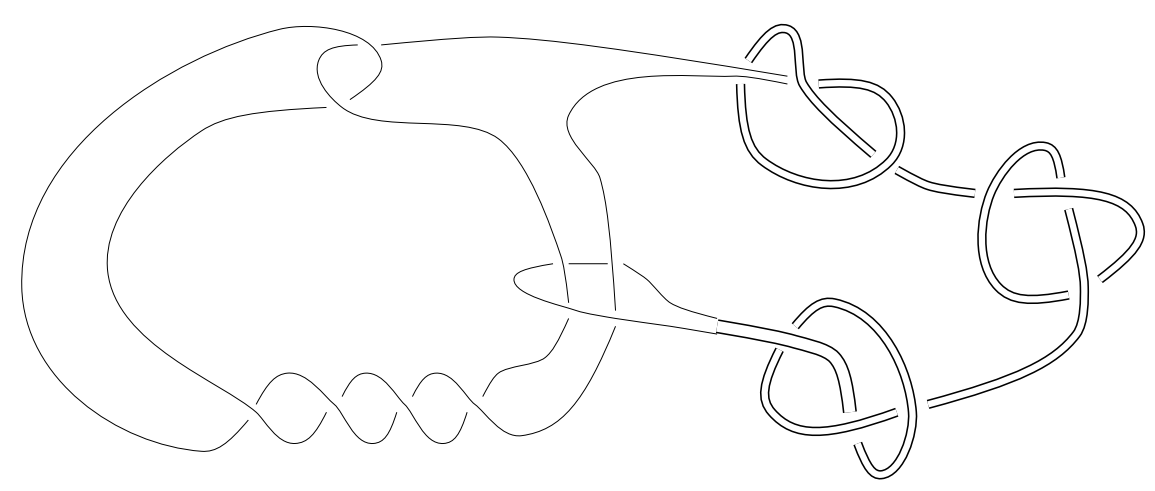

Figure 5: The knot $K$ with $\nu(K)=1$

$\nu(K)=1$. This knot is a satellite of a connected sum of three knots. We do not know an atoroidal knot with odd natural framing number.

We need one more technical tool. Let $K$ be a knot, and let $f: D \rightarrow S^{3}$ be any continuous map. We define a branched hole of $f$ to be a point $p \in D$ such that $f(p) \in K$ and such that $f$ maps the boundary of a small disk containing $p$ to some power of a meridian of $K$. So branched holes may just be transverse intersections of $f(D)$ with $K$, but they may also be essentially nontransverse. If all intersections of $f(D)$ with $K$ are branched holes, then we say $f$ is branched transverse.

Let $\rho_{n}: S^{1} \rightarrow S^{1}$ be the standard map of degree $n$. Then any branched transverse map $f: D \rightarrow S^{3}$ with $\left.f\right|_{\partial D}=K \circ \rho_{n}$ has at least one branched hole, by the loop theorem. More generally:

Lemma 3.1 Let $K_{1}, \ldots, K_{m}$ be nontrivial knots. Then any branched transverse map $f: D \rightarrow S^{3}$ with $\left.f\right|_{\partial D}=\left(K_{1} \# \ldots \# K_{m}\right) \circ \rho_{n}$ has at least $m$ branched holes.

Proof The proof is similar to, but even simpler than, the proof of Theorem 1.3. For any given compressing disk $f$ there exists a compressing disk $f^{\prime}$ with no more branched holes than $f$ and with only $m$ arcs (no closed curves) of intersection with a separating sphere. Then $f^{\prime}$ can be split into two disks, and the lemma follows inductively.

We are now ready to prove the main result of this section. Let $K$ be the knot indicated in Figure 5. 
Theorem 3.2 The framing function of $K$ satisfies $n(k)=|k|+2$ for $k \geqslant 2$ and $n(k)=|k|+4$ for $k \leqslant-4$. In particular, $\nu(K)=1$.

Proof It is easy to construct a compressing disk with 6 negative and 2 positive holes, so $n(k) \leqslant|k|+4$ for $k \leqslant-4$ (see Figure $6(\mathrm{a})$ ). It is also easy to construct a compressing disk with 1 negative and 3 positive holes (see Figure 6(b)), so
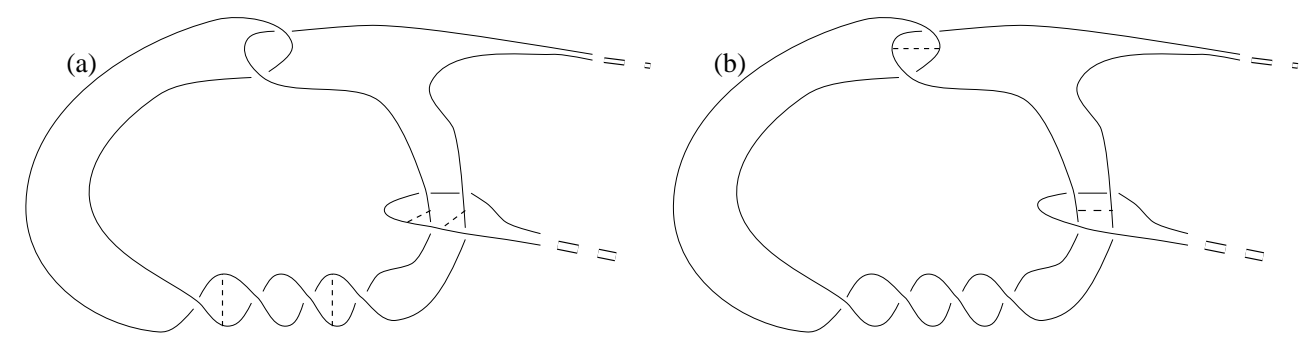

Figure 6: The two 'optimal' compressing disks of $K$

$n(k) \leqslant|k|+2$ for $k \geqslant 2$. We want to prove that these are in fact equalities, ie that every compressing disk of $K$ has at least one negative and two positive holes.

Consider the knotted solid torus $S$ containing $K$, with a meridinal curve $c$ on its boundary, as indicated in Figure 7. The core of $S$ is a connected sum of three trefoil knots.

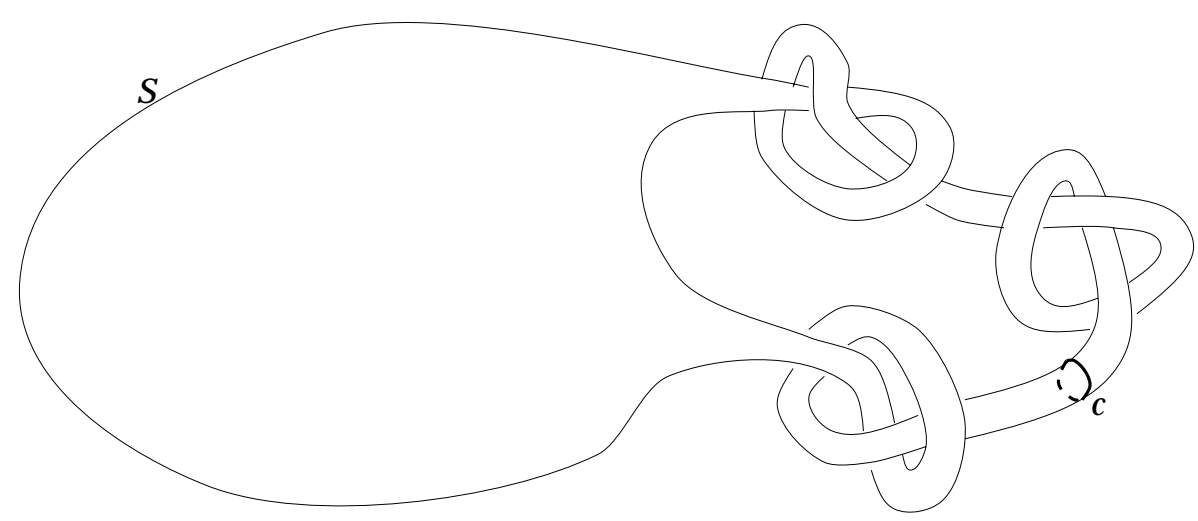

Figure 7: The solid torus $S$ containing $K$

Let $f: D \rightarrow S^{3}$ be a compressing disk transverse to $\partial S$ with $n(f)=n(k(f))$, ie $f$ has the minimal possible number of holes for its framing. Two compressing 
disks $f_{0}$ and $f_{1}$ are called isotopic if there is a homotopy $f_{t}(t \in[0,1])$ which is fixed on $\partial D$

$$
f_{t}:\left(D, D-f_{0}^{-1}(K)\right) \rightarrow\left(S^{3}, S^{3}-K\right) .
$$

We can assume that among all disks isotopic to $f, f$ has the minimal number of intersections points $f(D) \cap c$.

Lemma 3.3 The compressing disk does not intersect the curve $c$.

Proof of the Lemma Assume it does. Then we look at intersection lines $f(D) \cap \partial S$. Each such curve represents an element $(m, l) \in H_{1}(\partial S) \cong \mathbb{Z}^{2}$, with $(1,0)$ corresponding to a standard meridian of $S$. We can assume that there are no inessential curves, ie no curves representing $(0,0)$, because we can remove them by an isotopy of $f$. We call curves representing $(m, 0)(m \in \mathbb{Z} \backslash 0)$ meridinal curves, and all others except the trivial one longitudinal curves. Since $|f(D) \cap c|$ is assumed minimal, meridinal curves are disjoint from $c$, so there is at least one longitudinal curve. The preimages of longitudinal curves are disjoint embedded circles in $D$, and we let $\delta$ be an innermost one. Then $\delta$ bounds a disk $\Delta$ in $D$ such that $\left.f\right|_{\Delta}$ has only meridinal intersections with $\partial S$. These meridinal curves are noncontractible in $S-K$, and they have linking number 0 with $K$, so a disk in $\Delta$ bounded by the preimage of a meridinal curve contains at least one positive and one negative hole. By Lemma 3.1 there are at least three such meridinal curves. It follows that the framing disk has at least three positive and three negative holes, contradicting the hypothesis that the number of holes is minimal for its framing.

$S^{3} \backslash c$ is a solid torus; since, by Lemma 3.3, $f(D)$ is disjoint from $c$, we can lift $K$ and the compressing disk $f$ to its universal cover. This is an open, infinite solid cylinder, and thus homeomorphic to $\mathbb{R}^{3}$ (see Figure 8).

The preimage of $K$ under the covering space projection consist of a $\mathbb{Z}$-family of link components $\ldots, L_{-1}, L_{0}, L_{1}, L_{2}, \ldots$, but we simply take away the link components $\ldots, L_{-3}, L_{-2}, L_{-1}$ (see Figure 8 ). We denote by $f^{\prime}$ the lifting of the disk which sends $\partial D$ to $L_{1}$. $L_{1}$, regarded only as a closed curve, is noncontractible in $\mathbb{R}^{3} \backslash L_{2}$ and has linking number zero with $L_{2}$, so $f^{\prime}$ has at least one positive and one negative intersection with $L_{2}$. It follows that $f$ has at least one positive and one negative hole.

We can now also forget about the link components $L_{i}$ for $i \geqslant 2$, and only consider $L_{0}$ and $L_{1}$. 


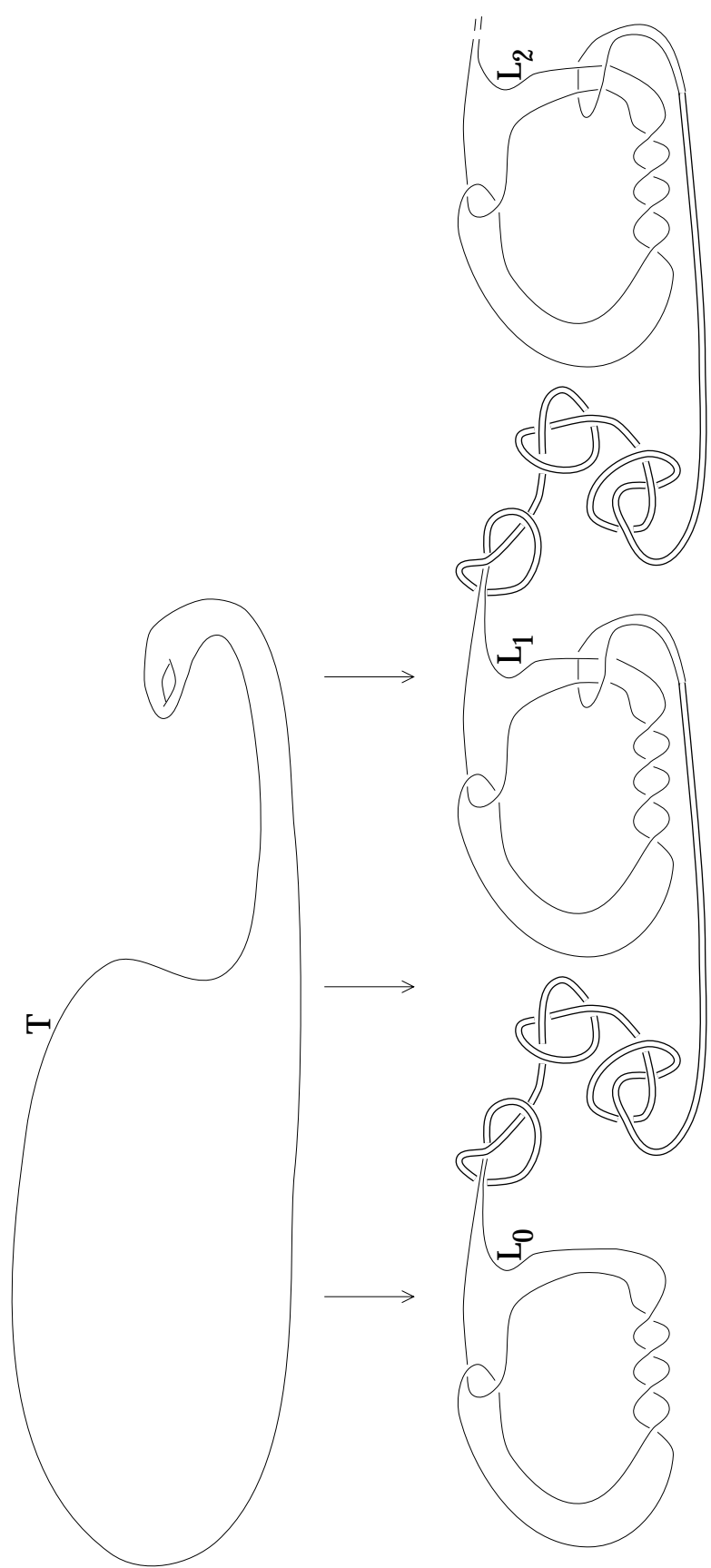

Figure 8: The universal cover of $S^{3}-c$ 
Lemma 3.4 The disk $f^{\prime}$ has at least one positive intersection, either with $L_{1}$ or with $L_{0}$.

Proof of the Lemma We embed a solid torus $T$ in $\mathbb{R}^{3}$ such that it contains $L_{0}$, as indicated. We can assume that $f^{\prime}$ has the minimal number of intersection lines with $\partial T$ among all compressing disks isotopic to $f^{\prime}$. There are two possibilities to consider. Either $f^{\prime}$ does not intersect $T$. Then again by a covering space argument we see that $f^{\prime}$ has at least two positive intersections with $L_{1}$. The other possibility is that $f^{\prime}$ has intersections with $\partial T$. The preimages of these intersection lines are disjoint embedded circles in $D$, and we denote by $\gamma$ an innermost one of them; $\gamma$ bounds a disk $\Gamma \subseteq D$. Recall that $f^{\prime}(\gamma)$ is assumed noncontractible in $\partial T$. So either $f^{\prime}(\gamma)$ is a power of the 0 -longitude of $T$, in which case $f^{\prime}(\gamma)$ has at least one positive and one negative intersection with $L_{1}$; or $f^{\prime}(\gamma)$ has nonzero linking number with the core of $T$, in which case the compressing disk must intersect $L_{0}$. Since $\operatorname{lk}\left(L_{1}, L_{0}\right)=0$, we have at least one positive and one negative intersection with $L_{0}$.

In summary, $f^{\prime}$ has at least one negative and one positive intersection with $L_{2}$, and at least one more positive intersection with $L_{1}$ or $L_{0}$, so $f$ has at least one negative and two positive holes. This completes the proof of Theorem 3.2.

The signature of a knot is always an even number. So in particular, the absolute value of the natural framing and the signature of a knot can definitely be different.

\section{The natural framing of torus knots}

In this section we prove that the natural framing of $T(p, q)$, the $(p, q)$-torus knot, is $-(p-1)(q-1)$. More precisely, we exhibit a compressing disk of $T(p, q)$ with $(p-1)(q-1)$ negative and no positive holes, and we prove that every compressing disk must have at least $(p-1)(q-1)$ negative holes. A very different, more constructive proof of the special case $q=2$ can be found in [4].

We start by reinterpreting the framing function. Let $K \subseteq S^{3}$ be an oriented knot, and let $G=\pi_{1}\left(S^{3} \backslash K\right)$ be the knot group. A positive or negative Wirtinger generator of $G$ is a path from the basepoint to the boundary of a tubular neighbourhood of $K$, then once around a meridian of the tubular neighbourhood according to the right or left hand rule respectively, and back along the 
first segment of path in the opposite direction. Let $x_{1}, \ldots, x_{m}$ be positive Wirtinger generators which together generate the knot group. Fix a path $\gamma$ from the boundary of a tubular neighbourhood of the knot to the basepoint. For $k \in \mathbb{Z}$ let $l_{k} \in G$ be the element represented by the path $\gamma^{-1}$ followed by the longitude with linking number $k$ with the knot followed by the path $\gamma$.

Lemma 4.1 Take $\sigma_{+}, \sigma_{-} \in \mathbb{N}$. Let $k=\sigma_{+}-\sigma_{-}$and $n=\sigma_{+}+\sigma_{-}$. Then the following statements are equivalent:

(i) There exists a compressing disk of $K$ with $\sigma_{+}$positive and $\sigma_{-}$negative holes.

(ii) The longitude $l_{k} \in G$ is represented by a word

$$
w_{1}^{-1} x_{i_{1}}^{\epsilon_{1}} w_{1} \ldots w_{n}^{-1} x_{i_{n}}^{\epsilon_{n}} w_{n},
$$

where each $w_{i}$ is a word in $\left\{x_{i}^{ \pm 1}\right\}$, each $\epsilon_{i}= \pm 1$, and $\sum \epsilon_{i}=k$.

Proof Suppose that (i) holds. By retracting the compressing disk to a onedimensional spine we see that the path $l_{k}$, which is the boundary of the compressing disk, is homotopic to a product of $\sigma_{+}$positive and $\sigma_{-}$negative Wirtinger generators. (ii) follows. Conversely, we can use a homotopy between a product of $\sigma_{+}$positive and $\sigma_{-}$negative Wirtinger generators and the path $l_{k}$ to construct a compressing disk with $\sigma_{+}$positive and $\sigma_{-}$negative holes.

Thus we can reinterpret the framing function $n_{K}: \mathbb{Z} \rightarrow \mathbb{N}$ as follows. For $k \in \mathbb{Z}$ we let

$$
n_{K}(k)=\min \left\{n \in \mathbb{N} \mid l_{k} \text { is represented by a word in the above form }\right\} .
$$

Note that $n_{K}(k)$ is independent of the choice of $\gamma$. Roughly speaking, we are trying to express the longitude with linking number $k$ as a shortest possible product of conjugates of the generators $x_{1}, \ldots, x_{m}$.

We call a finite presentation of a knot group in which all generators are Wirtinger generators a Wirtinger presentation. The Cayley graph $\Gamma$ associated to such a presentation has natural 'layers' corresponding to the elements' images under the natural map to $H_{1}\left(S^{3} \backslash K\right) \cong \mathbb{Z}$. Multiplying a given element of the knot group by any conjugate of a positive or negative Wirtinger generator corresponds to 'stepping one layer up' or 'down' respectively in $\Gamma$. There are $k$ conjugates algebraically in a word representing $l_{k}$. In the cases below, we shall be trying to use as few as possible, so we want to avoid taking steps 'down' in $\Gamma$, ie using negative conjugates. 
Consider the positive cone from the identity in $\Gamma$, the set of elements which may be written as a product of positive conjugates. We want to know how close $l_{k}$ gets to this cone as $k$ increases; if we can show that, however large $k$ gets, $l_{k}$ still requires steps down, we will obtain a negative upper bound on the natural framing of $K$. For the left-hand trefoil, for example, we prove that $l_{k}$ requires at least 2 steps down for any $k$, corresponding to 4 extra holes of a compressing disk; hence $n(k) \geqslant k+4$, and, since we know that for the trefoil $n(-2)=2$, we see $\nu(T(3,2))=-2$.

We shall show that $\nu(T(p, q))=-(p-1)(q-1)$ in two theorems, first for the case $q=2$ and then for $q \geqslant 2$. The first proof is really a degenerate case of the second, but we introduce the ideas used in both theorems in the simpler context of $q=2$ and leave the additional calculation to the second case, where it first becomes necessary.

Theorem 4.2 The natural framing of $T(p, 2)$ is $-(p-1)$; indeed, its framing function is given by $n(k)=(p-1)+|k+(p-1)|$.

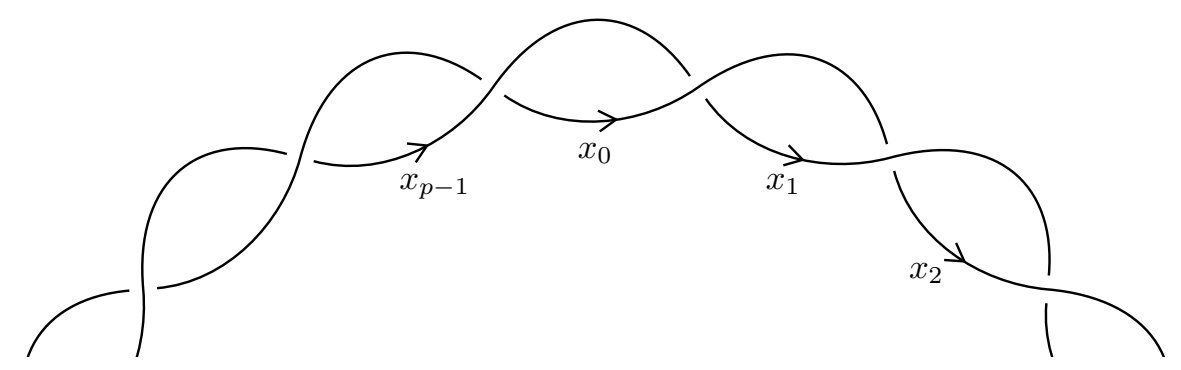

Figure 9: Generators of the knot group of the $(p, 2)$-torus knot

Proof Fix $p$. Draw $T(p, 2)$ in the usual way in a diagram with $p$ negative crossings and $p$-fold symmetry as in Figure 9. Label the overcrossing $\operatorname{arcs} x_{0}$, $x_{1}, \ldots, x_{p-1}$ clockwise around the diagram. Then the fundamental group of the complement is given by

$$
G=<x_{0}, x_{1}, \ldots, x_{p-1} \mid x_{0} x_{p-1}=\ldots=x_{2} x_{1}=x_{1} x_{0}>,
$$

and the word

$$
x_{0}^{k+p} x_{1}^{-1} x_{3}^{-1} \ldots x_{p-2}^{-1} x_{0}^{-1} x_{2}^{-1} \ldots x_{p-1}^{-1}
$$

represents the longitude $l_{k} \in G$. Notice that $n_{K}(-(p-1))=p-1$; this follows from the existence of the disk drawn in Figure 10, which has $p-1$ negative holes and no positive ones, and the fact that $n(k) \geqslant|k|$. 


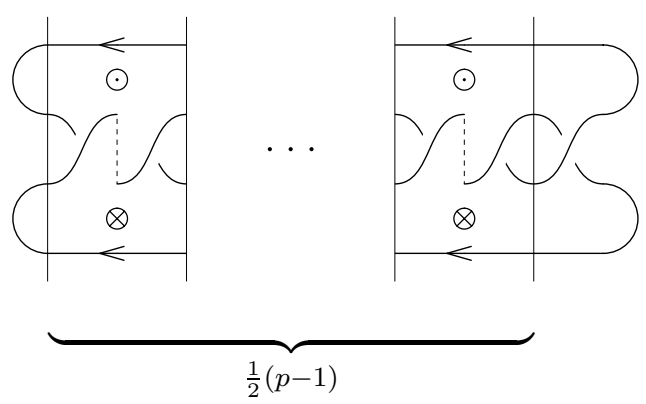

Figure 10: A disk with $p-1$ negative holes and no positive ones

Hence $n(k)=-k$ for $k \leqslant-(p-1)$. If we could show that, given $k$, there was a possibly larger integer (which instead we call $k$ ) such that $n(k) \geqslant k+2(p-1)$, we would know the entire framing function.

We aim to capture something of the geometry of the Cayley graph $\Gamma$ of $G$ with the above presentation, and show that $l_{k}$ is always 'hard to get to'. As an example, take $p=3$ and consider just a small portion of $\Gamma$, those vertices which may be written as the product of at most two (positive) generators. Three of these words coincide as group elements; apart from that, they are all different. This portion may be embedded in $\mathbb{R}^{3}$ as shown in Figure 11 .

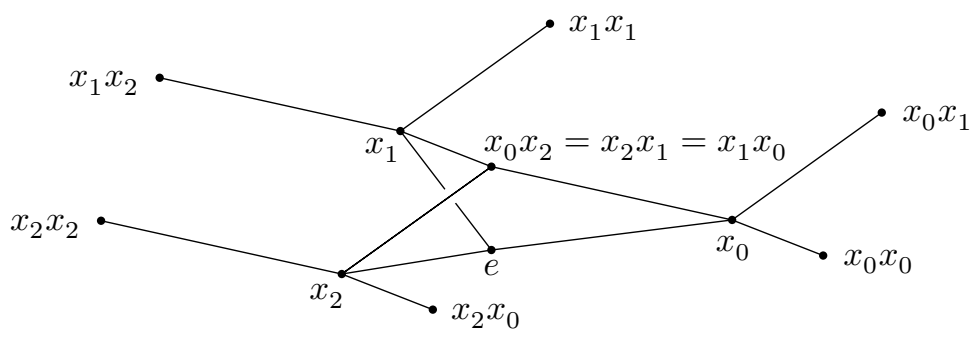

Figure 11: Embedding the Cayley graph of $G$ in $\mathbb{R}^{3}$

We may extend this embedding in a consistent way to the whole of $\Gamma$. (The exact meaning of 'consistent' is given implicitly by the definition of $\theta$ below.) Arranged thus, $\Gamma$ projects vertically down onto an infinite $p$-valent tree, which we can think of as the Cayley graph of

$$
I_{p}:={ }_{p} \mathbb{Z}_{2}=<0,1, \ldots, p-1 \mid 00=11=\ldots=(p-1)(p-1)=e>.
$$

(See below for a picture of this group's Cayley graph.) To realise this projection, we want a function $\theta: G \rightarrow I_{p}$. We shall find that $\theta$ is not a homomorphism. 
We first define $\theta$ on $X$, the set of words in the symbols $x_{0}, x_{1}, \ldots, x_{p-1}$ and their inverses:

$$
\theta: x_{i_{0}}^{\epsilon_{0}} x_{i_{1}}^{\epsilon_{1}} \ldots x_{i_{s-1}}^{\epsilon_{s-1}} \mapsto \prod_{j=0}^{s-1}\left(i_{j}+h_{j}\right)
$$

where each $i_{j} \in\{0,1, \ldots, p-1\}$, each $\epsilon_{j}= \pm 1$, and the $j$ th 'height' is

$$
h_{j}=\frac{\epsilon_{j}-1}{2}+\sum_{k=0}^{j-1} \epsilon_{k}
$$

(all addition modulo $p$ ). For example, when $p=3$, the word $x_{1} x_{2} x_{1} x_{0}^{-1} x_{0} x_{2}$ maps to 12 :

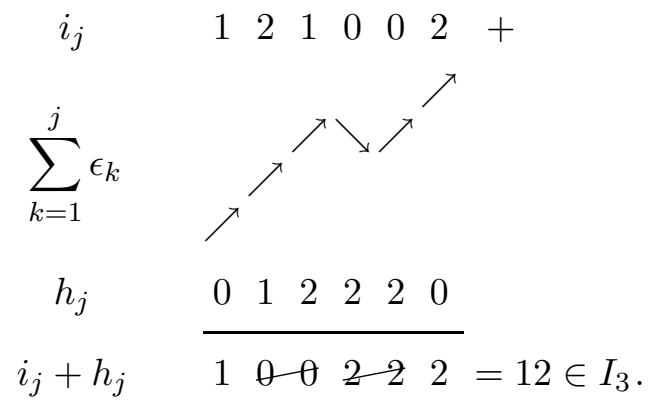

As this example shows, changing a word in $X$ by an elementary expansion or reduction (that is, insertion or deletion of a pair $x_{i} x_{i}^{-1}$ or $x_{i}^{-1} x_{i}$ ) does not change its image under $\theta$, since the two adjacent letters involved have the same index and height, and so map to a repeated element in $I_{p}$. Also, $x_{i+1} x_{i}$ maps to the identity at any height for any $i$, so changing our word in $X$ by a relator of $G$ leaves its image under $\theta$ unchanged. Hence $\theta$ is well-defined as a map from $G$ to $I_{p}$.

Suppose we have a word

$$
W=w_{1}^{-1} y_{1}^{\epsilon_{1}} w_{1} w_{2}^{-1} y_{2}^{\epsilon_{2}} w_{2} \ldots w_{n}^{-1} y_{n}^{\epsilon_{n}} w_{n}
$$

which represents $l_{k}$, where $n=k+2 t$ and each $y_{i} \in\left\{x_{0}, x_{1}, \ldots, x_{p-1}\right\}$. By adding small positive umbrellas, say by premultiplying $W$ by a power of $x_{0}$, we may assume that $k$ is a large positive multiple of $p$. (This is for notational convenience and to remove a special case later. Notice that $t$ is unaffected.) 
Since $W$ represents $l_{k}$,

$$
\begin{aligned}
& \theta(W)=\theta\left(\begin{array}{lllllllllll}
\left(x_{0} x_{0} \ldots\right. & x_{0} & )^{k / p+1} & x_{1}^{-1} & x_{3}^{-1} & \ldots & x_{p-2}^{-1} & x_{0}^{-1} & x_{2}^{-1} & \ldots & x_{p-1}^{-1}
\end{array}\right)
\end{aligned}
$$

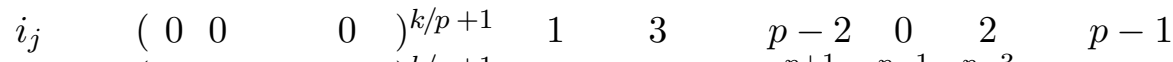

$$
\begin{aligned}
& h_{j} \quad\left(\begin{array}{llllllll}
0 & 1 & p-1
\end{array}\right)^{k / p+1} p-1 \quad p-2 \quad \frac{p+1}{2} \quad \frac{p-1}{2} \quad \frac{p-3}{2} \quad 0
\end{aligned}
$$

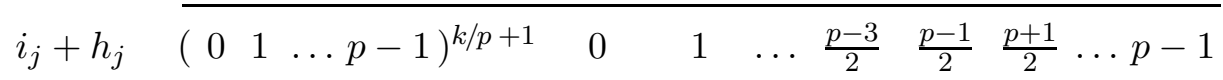

$$
\begin{aligned}
& =(01 \ldots(p-1))^{k / p+2} \in I_{p} .
\end{aligned}
$$

If the Cayley graph of $I_{p}$ with the above generators is drawn in the plane with edges consistently labelled 0 to $(p-1)$ anticlockwise round each vertex, we find that $\theta\left(l_{k}\right)$ turns sharp right at every step, and follows the boundary of one of the infinite complementary regions. For example, in the case $p=3$ and $k=0$ we have $\theta\left(l_{k}\right)=012012$, so the graph looks as in Figure 12 .

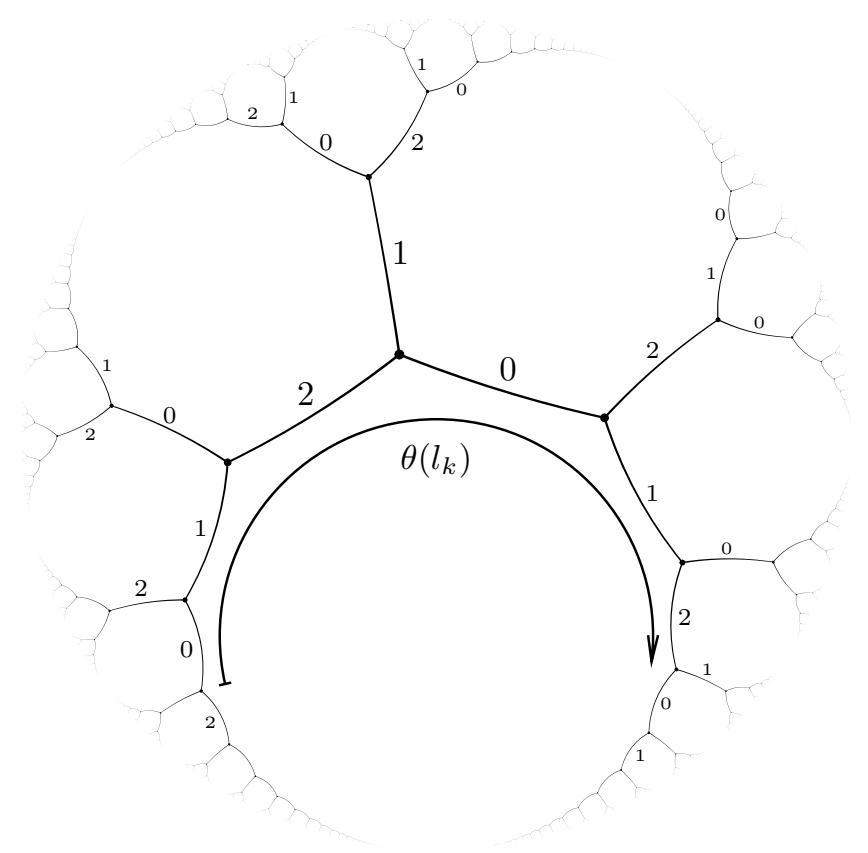

Figure 12: The Cayley graph of the group $I_{p}$

This suggests defining the angle $a(v)$ of a non-trivial reduced word $v$ in the 
symbols $\{0,1, \ldots, p-1\}$, which we think of as 'turn-right-ness', as

$$
a\left(i_{0} i_{1} \ldots i_{s-1}\right):=\sum_{j=1}^{s-1}\left(p-2 d_{j}\right),
$$

where $i_{j-1}+d_{j} \equiv i_{j}(\bmod p)$ and $d_{j} \in\{1,2, \ldots, p-1\}$.

Thus each step $i_{j-1}$ to $i_{j}$ contributes between $-p+2$ and $p-2$ to the angle, and the more often and more sharply a word 'turns right', the greater this angle. Define the angle of an element of $G$ as the angle of its reduced image under $\theta$. Since $\theta\left(l_{k}\right)$ turns sharp right $(k+2 p-1)$ times, the angle is $(k+2 p-1)(p-2)$; this will prove unusually high for its exponent sum. Notice that the angle of an element of $I_{p}$ is unchanged if we cycle the generators in its expression modulo $p$; that is,

$$
a\left(i_{0} i_{1} \ldots i_{s-1}\right)=a\left(\left(i_{0}+1\right)\left(i_{1}+1\right) \ldots\left(i_{s-1}+1\right)\right) .
$$

Let $W_{0}$ be the large power of $x_{0}$ we multiplied our original word by, and define inductively $W_{i}=W_{i-1} w_{i}^{-1} y_{i}^{\epsilon_{i}} w_{i}$, so $W_{n}=W$. We ask how the angles of these initial segments increase with $i$. Suppose we are stepping from $W_{i-1}$ to $W_{i}$. Since the angle is defined only for reduced words, we must cancel $\theta\left(W_{i}\right)$ down to its reduced form; we therefore pretend that $W_{i}$ is a word in the letters $\left\{x_{j}^{ \pm 1}\right\}$, and that $\theta\left(W_{i}\right)$ is a word in the generators of $I_{p}$ (the letter-by-letter image of $\left.W_{i}\right)$, and do this reduction in stages.

First, perform all elementary reductions on $\theta\left(W_{i-1}\right)$, the initial segment. The total angle for this segment is $a\left(W_{i-1}\right)$. Also, let $v^{\prime}$ and $v$ be the reduced forms of the words obtained from the segments $w_{i}^{-1}$ and $w_{i}$, respectively-notice that $v$ is the reverse of $v^{\prime}$ cycled by $\epsilon_{i}$, so $a\left(v^{\prime}\right)+a(v)=0$ (ie the angles for these segments cancel).

Next, consider the image of the whole segment $w_{i}^{-1} y_{i}^{\epsilon_{i}} w_{i}$. We currently have this in the form $v^{\prime} z v$, say, for some $0 \leqslant z<p$. This looks somewhat like a conjugate of $z$ in $I_{p}$, except that the generators in $v$ have been cycled by $\epsilon_{i}$ modulo $p$. The words $v$ and $v^{\prime}$ may already be trivial. If not, write $z^{\prime}$ for the last letter of $v^{\prime}$; then the first letter of $v$ is $\left(z^{\prime}+\epsilon_{i}\right)$. If $z$ equals $z^{\prime}$ or $\left(z^{\prime}+\epsilon_{i}\right)$, we may shorten $v$ and $v^{\prime}$ and change $z$, and still have an expression of the same form (and notice that two cancelling angles have been removed from the word). For example, in $I_{3},(0212) 0(0201)=(021) 2(201)=(02) 1(01)$. Thus $\theta\left(w_{i}^{-1} y_{i}^{\epsilon_{i}} w_{i}\right)$ cancels down to one of the four forms below. The only contributions to the total angles for these segments come from the steps either side of $z$, and so are as shown. 


$\begin{array}{rrrr}\text { case } \mathrm{A}: & \epsilon_{i}=+1 & \theta\left(w_{i}^{-1} y_{i}^{\epsilon_{i}} w_{i}\right)=z & a\left(w_{i}^{-1} y_{i}^{\epsilon_{i}} w_{i}\right)=0 \\ \text { B: } & +1 & v^{\prime} z v & -2 \\ \text { C: } & -1 & z & 0 \\ \text { D: } & -1 & v^{\prime} z v & 2\end{array}$

The only contribution to the angle of the whole word $\theta\left(W_{i}\right)$ we have not yet considered comes from the boundary between the images under $\theta$ (reduced as described) of $W_{i-1}$ and $w_{i}^{-1} y_{i}^{\epsilon_{i}} w_{i}$. If there is no cancellation at this position in the word, the contribution to the angle of $W_{i}$ from this junction is at most $(p-2)$. If the second part of $\theta\left(W_{i}\right)$ is fully absorbed by the first, the same is true, since each elementary reduction before the last removes two equal and opposite contributions to the angle, and the last removes just one, which is certainly at least $(-p+2)$. Notice that in cases $\mathrm{A}$ and $\mathrm{C}$ these are the only two possibilities. Notice also that we may assume that the first part is never completely absorbed by the second, by increasing the framing beforehand as described above.

This leaves the case where there is partial cancellation at this boundary. We have so far reached

$$
\underbrace{\ldots j k_{0} k_{1} \ldots k_{s-1}}_{\theta\left(W_{i-1}\right)} \underbrace{k_{s-1} \ldots k_{1} k_{0} l \ldots}_{\theta\left(w_{i}^{-1} y_{i}^{\epsilon_{i}} w_{i}\right)}
$$

say, where $j \neq l$. Cancelling this down, and ignoring pairs of equal and opposite angles which vanish in the process, we lose the angle contributions from $j k_{0}$ and $k_{0} l$, and gain instead that from $j l$. This changes the angle by $\pm p$. Notice, however, that if $j+1=k_{0}$ the angle must decrease.

In summary, then, changes from $W_{i-1}$ to $W_{i}$ of types $\mathrm{A}, \mathrm{B}$, or c increase the angle by at most $(p-2)$, and those of type D increase it by at most $(p+2)$. The angle of $l_{k}$ is unusually high for its framing $k$; to see this, let $\operatorname{esum}\left(W_{i}\right)$ be the sum of the exponents in $W_{i}$, ie the linking number of a path representing $W_{i}$ with $K$, and consider

$$
c\left(W_{i}\right):=a\left(W_{i}\right)-(p-2)\left(\operatorname{esum}\left(W_{i}\right)-1\right) .
$$

This is only increased in cases $\mathrm{C}$ and $\mathrm{D}$, by $(2 p-4)$ and $2 p$, respectively. Recall that $a\left(l_{k}\right)=(k+2 p-1)(p-2)$, so $c\left(W_{n}\right)=2 p(p-2)$, so we require at least $(p-2)$ CS or Ds and the same total number of As and Bs. Since our word $W$ consisted of $(k+t)$ positive conjugates and $t$ negative conjugates, we see that $t \geqslant p-2$. 
Suppose $t=p-2$. Then $c$ must increase by a full $2 p$ for each negative conjugate, so there are no steps of type c. Let $i$ be the first time we encounter a case other than A. All previous steps must have been multiplication by $x_{0}$ in $G$ (since the word must turn sharp right each time to avoid decreasing $c\left(W_{i}\right)$ ). Hence the word so far is just a power of $x_{0}$. But then at the boundary between $W_{i-1}$ and $w_{i}^{-1} y_{i}^{\epsilon_{i}} w_{i}$ we cannot increase $a$ by $p$, so $c$ must decrease. Hence $t>p-2$, so $n \geqslant k+2(p-1)$ and we are done.

Theorem 4.3 The natural framing of $T(p, q)$ is $-(p-1)(q-1)$; indeed, its framing function is given by $n(k)=(p-1)(q-1)+|k+(p-1)(q-1)|$.

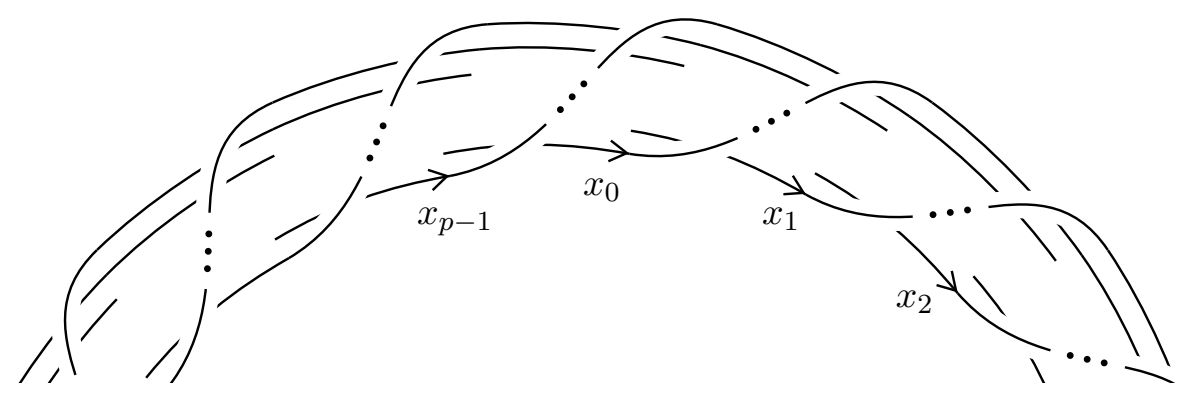

Figure 13: Generators of the knot group of the $(p, q)$-torus knot

Proof Fix $p$ and $q$ coprime with (say) $p>q$. From the diagram for $T(p, q)$ shown in Figure 13 we obtain the presentation

$G=<x_{0}, x_{1}, \ldots, x_{p-1} \mid x_{q-2} \ldots x_{0} x_{p-1}=\ldots=x_{q} \ldots x_{2} x_{1}=x_{q-1} \ldots x_{1} x_{0}>$

for $\pi_{1}\left(S^{3} \backslash K\right)$. Now $l_{k}$ is the group element represented by the word

$$
x_{0}^{k+p(q-1)} x_{1}^{-1} x_{2}^{-1} \ldots x_{q-1}^{-1} x_{q+1}^{-1} \ldots x_{2 q-1}^{-1} x_{2 q+1}^{-1} \quad \ldots \quad x_{p-1}^{-1} .
$$

It is less clear geometrically in this more general case that we can find a disk corresponding to the value $n(-(p-1)(q-1))=(p-1)(q-1)$ of the framing function of $T(p, q)$. Seen algebraically, however, we are trying to write the above word as a product of negative conjugates (again, all indices modulo $p$ ) when $k=-(p-1)(q-1)$. There are $(q-1) x_{0}^{-1} \mathrm{~s}$ in this expression. Conjugate each other inverse of a generator by $x_{0}^{-1}$ to the power of the number of $x_{0}^{-1} \mathrm{~s}$ after it; then elementary reduce the $x_{0} \mathrm{~s}$ and $x_{0}^{-1} \mathrm{~s}$. This gives an expression of the required form. For example, for $T(5,3)$, 


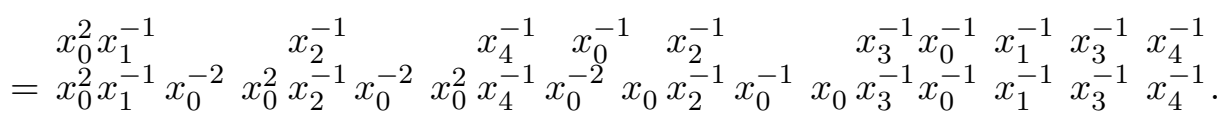

We would like a version of $\theta$ for this case; it must map a sequence of generators with gradually decreasing index of length $q$ (rather than 2 ) to the identity. This suggests defining $\theta$ from $G$ to

$$
{ }_{{ }_{p}} \mathbb{Z}_{q}:=<0,1, \ldots, p-1 \mid 0^{q}=1^{q}=\ldots=(p-1)^{q}=e>.
$$

As before, we first define $\theta$ on $X$, the set of words in the symbols $x_{0}, x_{1}, \ldots$, $x_{p-1}$ and their inverses:

$$
\theta: x_{i_{0}}^{\epsilon_{0}} x_{i_{1}}^{\epsilon_{1}} \ldots x_{i_{s-1}}^{\epsilon_{s-1}} \mapsto \prod_{j=0}^{s-1}\left(i_{j}+h_{j}\right)^{\epsilon_{i}},
$$

where each $i_{j} \in\{0,1, \ldots, p-1\}$, each $\epsilon_{j}= \pm 1$, and the height is again given by

$$
h_{j}=\frac{\epsilon_{j}-1}{2}+\sum_{k=0}^{j-1} \epsilon_{k}
$$

(all addition modulo $p$ ). Notice that this time we keep track of inverses of 0 , 1 , etc - in the involutary case, this was unnecessary.

Adjacent letters in a word in $X$ with the same index but of opposite sign have the same height, and so map to a cancelling pair in $*_{p} \mathbb{Z}_{q}$. Applying a relator of $G$ to a word $v$ in $X$ replaces, in $\theta(v)$, the $q$ th power of some generator of $*_{p} \mathbb{Z}_{q}$ by that of another. Therefore $\theta$ is well-defined as a map from $G$ to $*_{p} \mathbb{Z}_{q}$.

Suppose we take a framing disk. The longitude it follows is

$$
l_{k}=x_{0}^{k+p(q-1)} x_{1}^{-1} x_{2}^{-1} \ldots x_{q-1}^{-1} x_{q+1}^{-1} \ldots x_{2 q-1}^{-1} x_{2 q+1}^{-1} \ldots x_{p-1}^{-1}
$$

(all indices modulo $p$ ), where $k$ may be assumed to be a large positive multiple of $p$ by adding positive umbrellas as necessary. This maps under $\theta$ to

$$
\begin{aligned}
\theta\left(l_{k}\right) & =(01 \ldots(p-1))^{k / p+q-1} 0^{-(q-1)} 1^{-(q-1)} \ldots(p-1)^{-(q-1)} \\
& =(01 \ldots(p-1))^{k / p+q} .
\end{aligned}
$$

The 'angle' alone is now too small to give a tight bound on the natural framing of $T(p, q)$; we therefore introduce new ideas to make up the difference.

Since the negative powers of generators of $*_{p} \mathbb{Z}_{q}$ cancel so neatly in $\theta\left(l_{k}\right)$, we want this cancelling to 'score extra'. An element of $*_{p} \mathbb{Z}_{q}$ may be written in the 
standard form

$$
i_{0}^{e_{0}} i_{1}^{e_{1}} \ldots i_{s-1}^{e_{s-1}}
$$

with each $i_{j}$ a generator, $s$ minimal, and each $e_{j} \in\{1,2, \ldots, q-1\}$.

Adjacent $i_{j}$ s are then different. This allows us to define angle for such a standard form of a non-trivial word, much as before, by

$$
a\left(i_{0}^{e_{0}} i_{1}^{e_{1}} \ldots i_{s-1}^{e_{s-1}}\right):=\sum_{j=1}^{s-1}\left(p-2 d_{j}\right)
$$

where $i_{j-1}+d_{j} \equiv i_{j}(\bmod p)$ and $d_{j} \in\{1,2, \ldots, p-1\}$.

In addition, define the pseudo-exponent of $w \in G$ to be the sum of the exponents in the standard form of $\theta(w)$. The exponent sum of $w$ is the sum of exponents when $w$ is written in terms of the $x_{i}$ s. Then the excess exponent, $e(w)$, is defined to be the pseudo-exponent minus the exponent sum of $w$. Notice that $e(w)$ is a multiple of $q$. Finally, to register how close the excess exponent is to changing, define the internal angle $\iota$ of a word in standard form by

$$
\iota\left(i_{0}^{e_{0}} i_{1}^{e_{1}} \ldots i_{s-1}^{e_{s-1}}\right):=\sum_{j=0}^{s-1}\left(e_{j}-1\right) .
$$

Then the replacement we use for angle, which we call angle ${ }^{\prime}$ and denote $a^{\prime}$, is given by

$$
a^{\prime}(w):=a(w)-p \times \iota(w)+\frac{1}{q}(q-2) p \times e(w)
$$

Notice that $a^{\prime}$ is well-defined as a map from $G$ to $\mathbb{Z}$, since any element of $*_{p} \mathbb{Z}_{q}$ has a unique standard form. Angle' is not defined on $*_{p} \mathbb{Z}_{q}$, even though angle and internal angle are, because the excess exponent of an element is undefined. The angle' of $l_{k}$ is

$$
\begin{aligned}
a^{\prime}\left(l_{k}\right) & =(k+p q-1)(p-2)-0+\frac{1}{q}(q-2) p(k+p q-k) \\
& =(k-1)(p-2)+2 p((p-1)(q-1)-1),
\end{aligned}
$$

and this will turn out to be large enough to give the wanted tight upper bound on the natural framing.

Suppose we have a word

$$
W=w_{1}^{-1} y_{1}^{\epsilon_{1}} w_{1} w_{2}^{-1} y_{2}^{\epsilon_{2}} w_{2} \ldots w_{n}^{-1} y_{n}^{\epsilon_{n}} w_{n}
$$

which represents $l_{k}$, where $n=k+2 t$ and each $y_{i} \in\left\{x_{0}, x_{1}, \ldots, x_{p-1}\right\}$. As before, we may assume that $k$ is a large positive multiple of $p$. Let $W_{0}$ be the large power of $x_{0}$ which $W$ starts with, and define $W_{i}=W_{i-1} w_{i}^{-1} y_{i}^{\epsilon_{i}} w_{i}$. 
Although these are really group elements, we write them out literally in the letters $\left\{x_{i}^{ \pm 1}\right\}$ and pretend that they are just words. We use the literal version of $\theta$ to map them to $*_{p} \mathbb{Z}_{q}$. Only then do we do any simplification, ie elementary reduction and multiplication by $q$ th powers of generators. In this way, we can monitor the total angle ${ }^{\prime}$ of the result. We shall often calculate the angle ${ }^{\prime}$ of a segment of a word - this is again simply the angle' of the segment taken in isolation, not counting contributions to angle or internal angle from either end. The angle' of a set of segments of a word is the sum of the angle's for each segment.

We know that simplifying the $W_{i-1}$ segment of $W_{i}$ gives angle' $a^{\prime}\left(W_{i-1}\right)$. Consider next $w_{i}^{-1}$ and $w_{i}$. Taken as words, these map to, say,

and

$$
\begin{gathered}
v^{\prime}:=v_{0}^{e_{0}} v_{1}^{e_{1}} \ldots v_{s-1}^{e_{s-1}} \\
v:=\left(v_{s-1}+\epsilon_{i}\right)^{-e_{s-1}} \ldots\left(v_{1}+\epsilon_{i}\right)^{-e_{1}}\left(v_{0}+\epsilon_{i}\right)^{-e_{0}}
\end{gathered}
$$

(addition modulo $p$ ) respectively. We would like to show that these two expressions, taken together, contribute a total of 0 to $a^{\prime}$; however, we must convert them to standard form before we can measure this contribution. (By slight abuse of notation, we continue to call them $v^{\prime}$ and $v$ through the stages of this standardisation. We are effectively showing that $a^{\prime}(g)=-a^{\prime}\left(g^{-1}\right)$ for any $g \in G$.)

We may assume that each $e_{j}$ lies in $\{0,1, \ldots, q-1\}$ (by repeatedly multiplying by $v_{j}^{ \pm q}$ in $v^{\prime}$ and $\left(v_{j}+\epsilon_{i}\right)^{\mp q}$ in the corresponding place in $\left.v\right)$, and that none is 0 (by reducing $s$ ). To finish standardisation, we must, for each $j$, write the negative powers of $\left(v_{j}+\epsilon_{i}\right)$ as positive powers by multiplying by $\left(v_{j}+\epsilon_{i}\right)^{q}$ in the correct place in $v$. The pseudo-exponent then becomes $q s$, so $e\left(w_{i}^{-1}\right)+e\left(w_{i}\right)=$ $q s$. For each $j$ we have $e_{j} v_{j} \mathrm{~s}$ in $v^{\prime}$ and $\left(q-e_{j}\right)\left(v_{j}+\epsilon_{i}\right) \mathrm{s}$ in $v$, so the total internal angle, $\iota\left(v^{\prime}\right)+\iota(v)$, is $(q-2) s$. We already know the total angle is zerothe contribution from $v_{j} v_{j+1}$ in $v^{\prime}$ cancels with that from $\left(v_{j+1}+\epsilon_{i}\right)\left(v_{j}+\epsilon_{i}\right)$ in $v$-so the total angle ${ }^{\prime}$ for these two segments is, as wanted,

$$
a^{\prime}\left(v^{\prime}\right)+a^{\prime}(v)=0-p \times(q-2) s+\frac{1}{q}(q-2) p \times q s=0 .
$$

Next we consider the whole second segment of $\theta\left(W_{i}\right)$, namely $\theta\left(w_{i}^{-1} y_{i}^{\epsilon_{i}} w_{i}\right)$, where the images under $\theta$ of $w_{i}^{-1}$ and $w_{i}$ have already been simplified as above. Let $z^{\epsilon_{i}}$ be the image under $\theta$ of $y_{i}^{\epsilon_{i}}$. Perhaps $s=0$ in the above expressions. If so, $\theta\left(w_{i}^{-1} y_{i}^{\epsilon_{i}} w_{i}\right)=z^{\epsilon_{i}}$, which is already in standard form if $\epsilon_{i}=+1$, and may be written in the standard form $z^{q-1}$ when $\epsilon_{i}=-1$ making $e=q$ and $\iota=q-2$; both these expressions have angle 0 .

Consider the case $s>0$. By cycling the whole image modulo $p$, we may assume, for notational convenience, that $v_{s-1}$ and $\left(v_{s-1}+\epsilon_{i}\right)$ are 0 and 1 in some order. 
The only extra contributions to $a^{\prime}$ are those involving $z^{\epsilon_{i}}$. We watch how the angle' changes from $a^{\prime}\left(v^{\prime}\right)+a^{\prime}(v)$ to the angle' of $v^{\prime} z v$ after cancellation.

Suppose first that $\epsilon_{i}=+1$, so we find ...0z1 .. in the middle of $\theta\left(w_{i}^{-1} y_{i}^{\epsilon_{i}} w_{i}\right)$. If $z$ is not 0 or 1 , then $\iota$ and $e$ are unchanged and $a$ increases by $(p-2 z)+$ $(p-2(p-z+1))=-2$ (by definition, since $z$ and $(p-z+1)$ are both in $\{1,2, \ldots, p-1\})$. It follows that $a^{\prime}\left(w_{i}^{-1} y_{i}^{\epsilon_{i}} w_{i}\right)=-2$. If $z=0$ and $e_{s-1}<$ $q-1$, or if $z=1$ and $e_{s-1}>1$, we find $e$ unchanged, $\iota$ increased by 1 , and $a$ increased by $(p-2)$; again, $a^{\prime}\left(w_{i}^{-1} y_{i}^{\epsilon_{i}} w_{i}\right)=-2$. Finally, if $z=0$ and $e_{s-1}=q-1$, or if $z=1$ and $e_{s-1}=1$, we may pretend $\theta\left(w_{i}^{-1} y_{i}^{\epsilon_{i}} w_{i}\right)$ is really a (possibly trivial) conjugate of 1 (the first letter of $\theta\left(w_{i}\right)$ ) or 0 (the last letter of $\left.\theta\left(w_{i}^{-1}\right)\right)$ respectively with smaller $s$. This simplification removes two equal and opposite angles, reduces $e$ by $q$, and reduces $\iota$ by $(q-2)$, leaving $a^{\prime}$ unchanged. By induction, cancellation of this kind terminates in one of the cases already considered.

Suppose instead that $\epsilon_{i}=-1$, so we find $\ldots 1 z^{-1} 0 \ldots$ in the middle of the word $\theta\left(w_{i}^{-1} y_{i}^{\epsilon_{i}} w_{i}\right)$. We may write the central $z^{-1}$ as $z^{q-1}$, since this increases $\iota$ by $(q-2)$ and $e$ by $q$, leaving $a^{\prime}$ unchanged. Now, if $z$ is not 0 or 1 , then $\iota$ and $e$ are unaltered and the angle $a$ increases by $(p-2(z-1))+(p-2(p-z))=2$ (as above, since now $(z-1)$ and $(p-z)$ are in $\{1,2, \ldots, p-1\})$. In this case $a^{\prime}\left(w_{i}^{-1} y_{i}^{\epsilon_{i}} w_{i}\right)=2$. If $z=1$ and $e_{s-1}>1$ (or if $z=0$ and $e_{s-1}<$ $q-1$ ) then a block of $q 1$ s (or 0 s) may be removed from the centre, leaving $\ldots 1^{e_{s-1}-1} 0^{q-e_{s-1}} \ldots$ (or $\ldots 1^{e_{s-1}} 0^{q-e_{s-1}-1} \ldots$ ). In this middle segment, $e$ is reduced by $q, \iota$ is reduced from $(q-2)+(q-2)$ to $(q-3)$, and $a$ is increased by $(p-2)$; again, $a^{\prime}\left(w_{i}^{-1} y_{i}^{\epsilon_{i}} w_{i}\right)=2$. Finally, if $z=1$ and $e_{s-1}=1$, or if $z=0$ and $e_{s-1}=q-1$, we may pretend that $\theta\left(w_{i}^{-1} y_{i}^{\epsilon_{i}} w_{i}\right)$ is really a (possibly trivial) conjugate of $0^{q-1}$ (the first $(q-1)$ letters of $\left.\theta\left(w_{i}\right)\right)$ or $1^{q-1}$ (the last $(q-1)$ letters of $\left.\theta\left(w_{i}^{-1}\right)\right)$ respectively with smaller $s$. Again, the simplification removes two equal and opposite angles, reduces $e$ by $q$, and reduces $\iota$ by $(q-2)$, and therefore leaves $a^{\prime}$ unchanged; hence, by induction, cancellation of this kind terminates in one of the cases already considered.

Thus we have essentially the same set of cases as before:

\begin{tabular}{|c|c|c|c|}
\hline case A: & $\epsilon_{i}=+1$ & $\theta\left(w_{i}^{-1} y_{i}^{\epsilon_{i}} w_{i}\right)=z$ & $a^{\prime}\left(w_{i}^{-1} y_{i}^{\epsilon_{i}} w_{i}\right)=0$ \\
\hline B: & +1 & $v^{\prime} z v$ & -2 \\
\hline C: & -1 & $z^{q-1}$ & 0 \\
\hline D: & -1 & $v^{\prime} z^{q-1} v$ & 2 \\
\hline
\end{tabular}

Finally, there is the junction between $\theta\left(W_{i}\right)$ (say ending in $\left.x\right)$ and $\theta\left(w_{i}^{-1} y_{i}^{\epsilon_{i}} w_{i}\right)$ (say beginning with $y$ ) to consider, where each part here has been written in 
standard form as described above. The total angle ${ }^{\prime}$ (ie the sum of the angle's) of these segments, taken separately, is $a^{\prime}\left(W_{i}\right)$ or $a^{\prime}\left(W_{i}\right) \pm 2$, depending on the form of $\theta\left(w_{i}^{-1} y_{i}^{\epsilon_{i}} w_{i}\right)$. If $x \neq y$, the juxtaposition of these strings is already in standard form; then $\iota$ and $e$ remain the same and $a$ increases by at most $(p-2)$, so $a^{\prime}$ increases by at most $(p-2)$.

Suppose, then, that $x=y$. Some cancelling may occur at the boundary - if it does, we continue to call the shorter (standard form) words whose product is $\theta\left(W_{i+1}\right)$ 'the first word' and 'the second word', even though they change. Say the first word ends in $\ldots x^{\prime} x^{j}$, where $x^{\prime} \neq x$. (We may always assume that we can find such an $x^{\prime}$, by having premultiplied $W$ by a sufficiently high power of $x_{0}$ before we started.) The second may begin with $x^{k} x^{\prime \prime}$, where $x \neq x^{\prime \prime}$, or may simply be of the form $x^{k}$. Hence we have

$$
\text { either } \ldots x^{\prime} x^{j} x^{k} x^{\prime \prime} \ldots \text { or } \ldots x^{\prime} x^{j} x^{k}
$$

at the boundary. Since the words are in standard form, $j$ and $k$ are both in $\{1,2, \ldots, q-1\}$. Compare $(j+k)$ with $q$ :

$j+k<q$ : The juxtaposition of these strings is $\ldots x^{\prime} x^{j+k} x^{\prime \prime} \ldots$, which is already in standard form, so $\iota$ increases by 1 and $a^{\prime}$ drops by $p$.

$j+k>q$ : The juxtaposed strings cancel to $\ldots x^{\prime} x^{j+k-q} x^{\prime \prime} \ldots$, so $e$ decreases by $q$, but $\iota$ drops by $(q-1)$, so $a^{\prime}$ increases by $p$.

$j+k=q: \quad x^{j}$ and $x^{k}$ cancel, reducing $e$ by 1 and $\iota$ by $(q-2)$, and hence leaving $a^{\prime}$ unchanged. Then one of the following holds.

(i) There is no $x^{\prime \prime}$, so the word becomes $\ldots x^{\prime}, a$ drops by at least $(-p+$ 2 ), and $e$ and $\iota$ are unchanged, so $a^{\prime}$ increases by at most $(p-2)$.

(ii) $x^{\prime} \neq x^{\prime \prime}$, so the string becomes $\ldots x^{\prime} x^{\prime \prime} \ldots, e$ and $\iota$ are unchanged, and $a$ (and hence $a^{\prime}$ ) changes by either $+p$ or $-p$ (certainly $-p$ if $\left.x^{\prime}+1=x\right)$.

(iii) $x^{\prime}=x^{\prime \prime}$, and we can induct with shorter words.

The largest possible change to $a^{\prime}$ from this boundary is therefore $p$, as before, so we find that $a^{\prime}\left(W_{i+1}\right)-a^{\prime}\left(W_{i}\right)$ is at most $(p-2),(p-2), p$, and $(p+2)$ in the cases A to D, respectively. But $a^{\prime}\left(l_{k}\right)=(k-1)(p-2)+2 p((p-1)(q-1)-1)$, so if $t$ is the number of negative conjugates in $W$ we have

$$
\begin{array}{ll}
(k-1)(p-2)+t(p-2)+t(p+2) \geqslant(k-1)(p-2)+2 p((p-1)(q-1)-1) \\
\Rightarrow \quad t \geqslant(p-1)(q-1)-1 .
\end{array}
$$

Geometry and Topology, Volume 2 (1998) 
Suppose $t=(p-1)(q-1)-1$. Then there are no steps of type c, since each negative conjugate must increase $a^{\prime}$ by the full $(p+2)$. Any initial steps of type A must be multiplication of the word in $G$ by $x_{0}$, since $a^{\prime}$ must increase by $(p-2)$ each step and the only way to do this is to increase $a$ by the largest possible amount. But then the first step of type B or D must reduce $a^{\prime}$ by $p$ at the boundary between $W_{i-1}$ and $w_{i}^{-1} y_{i}^{\epsilon_{i}} w_{i}$ (see the case labelled (ii) above), which is a contradiction.

Hence $t \geqslant(p-1)(q-1)$, and we are done.

The theorem yields many other knots for which the absolute values of the natural framing number and the signature are different. For instance, if $K$ is the $(3,7)$ torus knot then $\nu(K)=-12$ and, according to [7], $\sigma(K)=8$. In this case, $|\nu(K)|>|\sigma(K)|$. As M Lackenby [6] has pointed out, one can use this fact to construct non-prime knots with $|\nu(K)|<|\sigma(K)|$. For instance, for $K=T(3,7) \# T(2,-13)$ we have $\nu(K)=12-12=0$ and $\sigma(K)=-8+12=4$.

If our conjecture on the natural framing of $\frac{4 m l+1}{2 l}$-two bridge knots is true, then we obtain many more knots $K$ with $|\nu(K)| \neq|\sigma(K)|$. For instance, for $m=l=2$ we would have that the knot $7_{4}$ has natural framing -4 , whereas its signature is 2 (see the table in [1]). Still, it is reasonable to expect that the natural framing number has some properties similar to the signature:

Questions (1) Is $\nu(K)$ even if $K$ is atoroidal, or if $K$ is a two-bridge knot?

(2) Is the natural framing number of slice knots always zero? Is the natural framing number a concordance-invariant?

(3) Is $2 u(K) \geqslant|\nu(K)|$, where $u$ is the unknotting number? Is $|\nu(K)| \geqslant|\sigma(K)|$ if $K$ is a prime knot? Is $|\nu(K)| \leqslant 2 g(K)$, where $g(K)$ is the four-ball genus? We conjecture that the answer to all three questions is No.

(4) Is there a finite algorithm to compute the natural framing number of a given knot? What about two-bridge knots?

\section{A table}

The following table contains all we know about the natural framing numbers of prime knots with up to seven crossings. For each of these knots it states the range within which the natural framing could possibly lie, and the value which we conjecture. For the convenience of the reader, we have added columns for invariants which may be related to the natural framing: the signature (taken 
from [1]), the blackboard-framing of an alternating diagram (see [8]), and the unknotting number (taken from [5]).

\begin{tabular}{cccccc} 
knot & \multicolumn{2}{c}{ natural framing } & signature & $\begin{array}{c}\text { alternating } \\
\text { diagram }\end{array}$ & $\begin{array}{c}\text { unknotting } \\
\text { number }\end{array}$ \\
$3_{1}$ & 2 & & -2 & 3 & 1 \\
$4_{1}$ & 0 & & 0 & 0 & 1 \\
$5_{1}$ & 4 & & -4 & 5 & 2 \\
$5_{2}$ & {$[0,2]$} & 2 & -2 & 5 & 1 \\
$6_{1}$ & 0 & & 0 & 2 & 1 \\
$6_{2}$ & {$[0,2]$} & 2 & -2 & 2 & 1 \\
$6_{3}$ & 0 & & 0 & 0 & 1 \\
$7_{1}$ & 6 & & -6 & 7 & 3 \\
$7_{2}$ & {$[0,2]$} & 2 & -2 & 7 & 1 \\
$7_{3}$ & {$[0,4]$} & 4 & -4 & 7 & 2 \\
$7_{4}$ & {$[0,4]$} & 4 & -2 & 7 & 2 \\
$7_{5}$ & {$[0,4]$} & 4 & -4 & 7 & 2 \\
$7_{6}$ & {$[0,2]$} & 2 & -2 & 3 & 1 \\
$7_{7}$ & 0 & & 0 & 1 & 1
\end{tabular}

Proof $3_{1}, 5_{1}$ and $7_{1}$ are torus knots. $4_{1}$ and $6_{1}$ belong to the family of twist knots with natural framing $0.6_{3}$ is amphichiral. It is easy to find compressing disks of the knots $5_{2}, 7_{2}, 7_{3}, 7_{4}, 7_{5}$ and $7_{6}$ with no ribbons, no negative clasps, only the appropriate number of positive clasps. (Note that the values for $5_{2}, 7_{2}$ and $7_{4}$ have been conjectured in section 1.) The only cases where the compressing disks are not easy to imagine are $6_{2}$ and $7_{7}$.
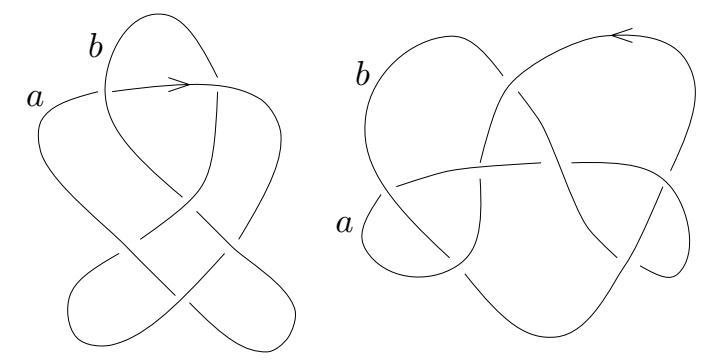

Figure 14: The knots $6_{2}$ and $7_{7}$

We see that $6_{2}$ has longitude

$$
l=a^{*} b A B a B A b A B a b^{*} a B A b A B a B A b
$$


(where we write $A$ and $B$ for $a^{-1}$ and $b^{-1}$, respectively); we can write this as

$$
a^{2} b A \underline{B} a B A b A \underline{B} a b^{-2} a \underline{B} A b A B a \underline{B} A b \text {. }
$$

If we leave out the four underlined letters we obtain the trivial word. This proves that $n(4)=4$. On the other hand, we can write the longitude as

$$
a^{3} \underline{b} A B a \underline{B} A b A B a b^{1} \underline{a} B A b A B a \underline{B} A b,
$$

so $n(0) \leqslant 4$. It follows that $0 \leqslant \nu\left(6_{2}\right) \leqslant 2$.

The knot $7_{7}$ has longitude

$$
l=a^{*} b A B a B A b a B a b A b a B A b A B a b^{*} a B A b A B a b A b a B a b A B a B A b .
$$

We can write this as

$$
a^{4} b A B a B A b \underline{a} B a b A b a B A \underline{b} A B \underline{a} b^{2} \underline{a} B A \underline{b} A B a b A b a B \underline{a} b A B a B A b .
$$

Leaving out the six underlined letters yields the trivial word, which proves that $n(-6)=6$. Also, we can write the longitude as

$$
a^{-6} b \underline{A} B a B \underline{A} b a \underline{B} a b \underline{A} b a \underline{B} A b A \underline{A} a b^{-6} a \underline{B} A b A \underline{B} a b \underline{A} b a \underline{B} a b \underline{A} B a B \underline{A} b,
$$

so $n(12)=12$. It follows that $\nu\left(7_{7}\right)=0$.

Remark These compressing disks were found using box-diagrams (see [4]).

Acknowledgements The authors thank their respective PhD advisors Brian Sanderson and Colin Rourke for their help and enthusiasm. M.T.G. was sponsored by EPSRC, B.W. by a University of Warwick Graduate Award.

\section{References}

[1] G Burde, H Zieschang, Knots, deGruyter (1985)

[2] $\mathbf{J} \mathbf{H}$ Conway, An enumeration of knots and links, and some of their algebraic properties, from: "Computational Problems in Abstract Algebra", Pergamon Press (1969) 329-385

[3] C A Giller, A family of links and the Conway calculus, Trans. Amer. Math. Soc. 270 (1982), 75-109

[4] M T Greene, B Wiest, A natural framing of knots III, Warwick preprint (December 1996) or http://www.maths.warwick.ac.uk/ 'bertold

[5] R Kirby, Problems in Low-dimensional topology (1996), available electronically from http://maths.berkley.edu/ ${ }^{k i r b y}$ or http://www. maths. warwick.ac.uk/ $\mathrm{cpr}$ 
[6] M Lackenby, personal communication

[7] R A Litherland, Signatures of iterated torus knots, from: "Topology of lowdimensional manifolds", Springer Lecture Notes in Mathematics 722 (1977), $71-84$

[8] K Murasugi, Jones polynomial and classical conjectures in knot theory II, Math. Proc. Camb. Phil. Soc. 102 (1987), 317-318

[9] E Pannwitz, Eine Elementargeometrische Eigenschaft von Verschlingungen und Knoten, Mathematische Annalen 108 (1933)

[10] D Rolfsen, Knots and Links, Publish or Perish (1976)

[11] $\mathbf{H}$ Whitney, The singularities of a smooth $n$-manifold in $(2 n+1)$-space, Annals of Math. 45 (1944), 247-293 